\title{
Assessing the transferability of support vector machine model for estimation of global solar radiation from air temperature
}

\author{
Ji-Long Chen ${ }^{\text {a,b,c,d }}$, Guo-Sheng Li ${ }^{c, d}$, Bei-Bei Xiao ${ }^{\text {e,f,g }}$, Zhao-Fei Wen ${ }^{\text {a,b }}$, Ming-Quan Lv ${ }^{\text {a,b }}$, Chun-Di Chen ${ }^{\text {a,b }}$, \\ Yi Jiang ${ }^{\mathrm{a}, \mathrm{b}}$, Xiao-Xiao Wang ${ }^{\mathrm{a}, \mathrm{b}}$, Sheng-Jun $\mathrm{Wu}^{\mathrm{a}, \mathrm{b}, *}$
}

${ }^{a}$ Chongqing Institute of Green and Intelligent Technology, Chinese Academy of Sciences, Chongqing 401122, China

${ }^{\mathrm{b}}$ Key Laboratory on Water Environment of Reservoir Watershed, Chinese Academy of Sciences, Chongqing 401122, China

${ }^{\mathrm{c}}$ Institute of Geographic Sciences and Natural Resources Research, Chinese Academy of Sciences, Beijing 100101, China

${ }^{\mathrm{d}}$ Laboratory of Land Surface Pattern and Simulation, Institute of Geographical Sciences and Natural Resources Research, Chinese Academy of Sciences, China

${ }^{\mathrm{e}}$ Chongqing Institute of Surveying and Planning for Land Resources and Housing, Chongqing 400020, China

${ }_{\mathrm{f}}^{\mathrm{f}}$ National Engineering Technology Research Center for Remote Sensing Applications, Chongqing Research Center, Chongqing 400020, China

${ }^{\mathrm{g}}$ Chongqing Xinrong Institute of Surveying Technology for Land and Housing, Chongqing 400020, China

\section{A R T I C L E I N F O}

\section{Article history:}

Received 3 July 2014

Accepted 1 October 2014

Available online 21 October 2014

\section{Keywords:}

Global solar radiation

Support vector machine

Air temperatures

Distance

Altitude

\begin{abstract}
A B S T R A C T
Exploring novel methods for estimation of global solar radiation from air temperature has been being a focus in many studies. This paper evaluates the transferability of support vector machines (SVM) for estimation of solar radiation in subtropical zone in China. Results suggest that solar radiation at one site (estimation site) could be well estimated by SVM model developed at another site (source site). The accuracy of estimation is affected by the distance and temperature difference between two sites, and altitude of source site. Higher correlations between RMSE of SVM and distance, and temperature differences are observed in northeastern region, increasing the reliability and confidence of SVM model developed at nearby stations. While lower correlations between RMSE and distance, and temperature differences are observed in southwest plateau region. When the altitude of estimation site is lower than $1200 \mathrm{~m}$, RMSE show logarithm relationship with altitude of source sites where the altitude are lower than that of estimation site. Otherwise, RMSE show linearly relationship with altitude of source sites where the altitude are higher than $200 \mathrm{~m}$ but lower than that of the estimation site. This result suggests that solar radiation could be also estimated using SVM model developed at the site with similar but lower altitude. Based on these results, a strategy that takes into account the climatic conditions, topography, distance, and altitude for selecting a suitable source site is presented. The findings can guide and ease the appropriate choice of source sites for estimation of solar radiation at estimation site.
\end{abstract}

(c) 2014 Elsevier Ltd. All rights reserved.

\section{Introduction}

The importance of global solar radiation in ecology, agriculture, environment and the associated researches has been well documented [1-3]. However, due to the cost of measuring equipment and its difficult maintenance and calibration, lack of sufficient solar radiation data has been reported in many countries like USA [4,5], United Kingdom [6], Egypt [7], India [8], France [9], Greece [10], Italy [11] and China [12-14]. On the contrary, air temperature is routinely measured at most meteorological stations. In this context, great efforts have been made to estimate

\footnotetext{
* Corresponding author at: Chongqing Institute of Green and Intelligent Technology, Chinese Academy of Sciences, Chongqing 401122, China. Tel.: +86 0236593 5918.

E-mail address: chenjilong@cigit.ac.cn (S.-J. Wu).
}

solar radiation using air temperature $[15,16]$. Hargreaves and Samani [15] proposed a model ( $\mathrm{H}-\mathrm{S}$ model) using air temperature range, several modified versions were developed and validated in many places around the world [17-20]. Although some authors claimed that their new versions outperformed the original model, this may not always be the cases in many studies even when other commonly measured meteorological variables are taken into account [4,13,21,22]. Bristow and Campbell [16] developed a model ( $\mathrm{B}-\mathrm{C}$ model) as exponential function of temperature range. Numerous modifications centered on tuning the parameters of B-C model have been made [23-25]. However, many evaluations suggested that such modifications were generally not effective and yielded little or no improvement [26-28].

Accurate estimation of solar radiation has been being a major goal for solar energy practitioners, climatologists and all concerned scientists [29]. It seems that $\mathrm{H}-\mathrm{S}, \mathrm{B}-\mathrm{C}$ models and their revised 


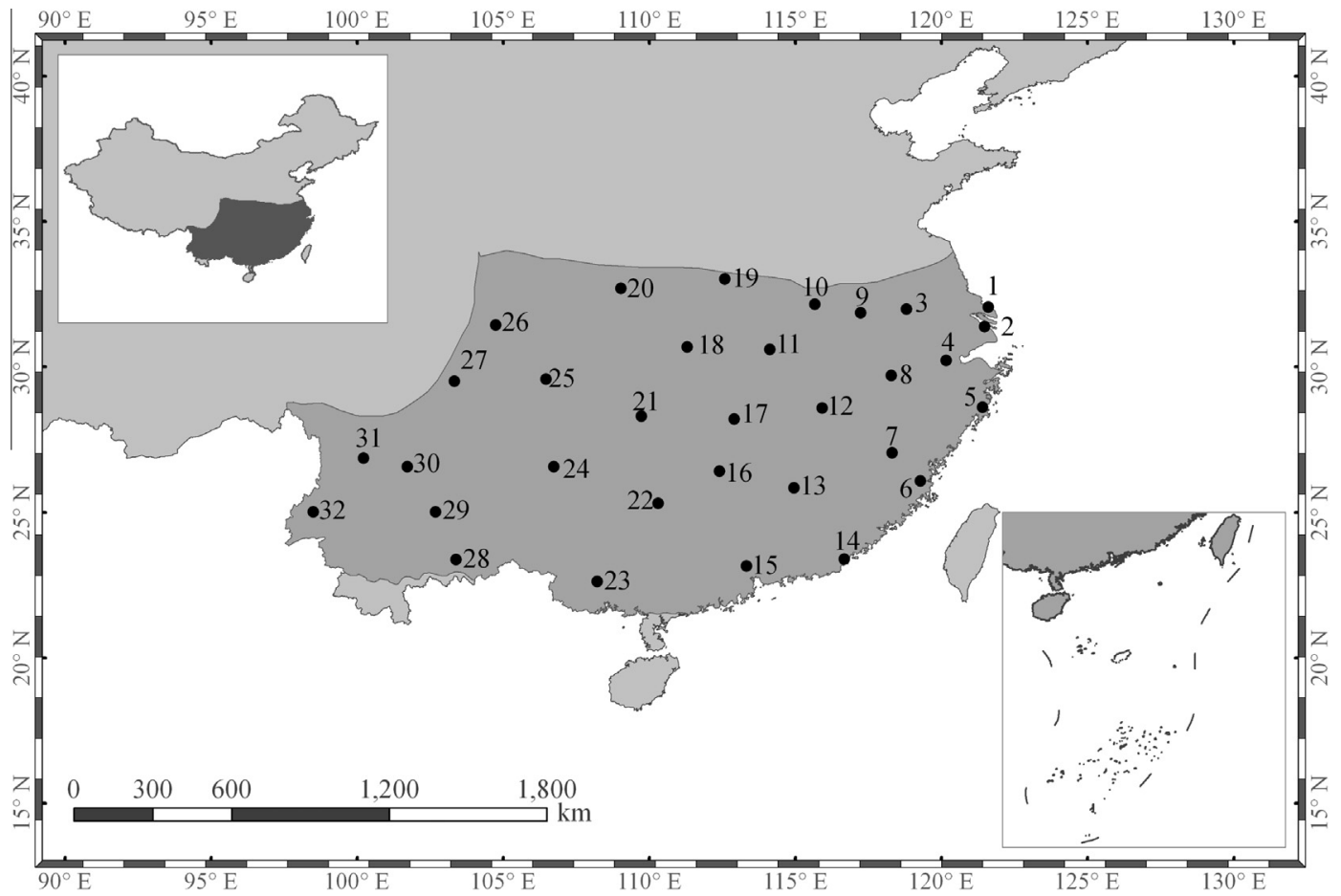

Fig. 1. Location of the studied meteorological stations in China's subtropical zone (stations are numbered in compliance with Table 1).

versions, as well as other empirical models, have far overreached their predictive limits. Therefore, exploring new methods has became the focus of many studies in recent years [30-39].

Recently, as the development of computational technology and sophisticated statistical methods, a novel machine learning method, support vector machine (SVM), has been widely applied in computer [40], environment [41,42] and hydrology researches [43], and proven to be a robust algorithm in classification [44], regression [45,46] and forecasting [47]. Many studies proved that SVM is superior to Neural Network and traditional statistical models [48-50]. Despite successes in many fields, there is not any application in estimation of solar radiation until our work investigated the feasibility of SVM using air temperatures at Chongqing, China [3]. While it is still needed to research further to solve the problem how to apply SVM model to the site where no solar radiation data is available. One possible alternative is to substitute training data from nearby representative meteorological stations. It was proposed by Allen et al. [51] as a possible way of replacing missing data when calculating daily evapotranspiration. However, there is lack of explicit guidance on appropriate choice of the representative stations, which may result in arbitrariness in choosing a source site that provides data for constructing SVM model for solar radiation estimation at the estimation site. Thus, the transferability of SVM model is investigated.

The present study is carried out in China's subtropical zone which plays an important role in ecological, agricultural and climate researches in the world. However, there are only 32 meteorological stations measuring global solar radiation. On the contrary, more than 700 stations have records of air temperatures, offering an important alternative to deriving solar radiation due to the widely availability of temperature data. Some works validated the temperature-based models for this area [52-54], and a few revised models were developed $[13,52]$. However, these new versions were found to give similar performances with the original ones at many sites [13,52,55]. Apart from model accuracy, one major limitation is that calibration of those empirical models is constrained by unavailability of solar radiation data, limiting
Table 1

Detail information of the studied meteorological stations.

\begin{tabular}{|c|c|c|c|c|}
\hline Site ID & Site name & Longitude (E) & Latitude $(\mathrm{N})$ & Altitude (m) \\
\hline 1 & Lvshi & 121.60 & 32.07 & 5.5 \\
\hline 2 & Shanghai & 121.48 & 31.40 & 6.0 \\
\hline 3 & Nanjing & 118.80 & 32.00 & 7.1 \\
\hline 4 & Hangzhou & 120.17 & 30.23 & 41.7 \\
\hline 5 & Hongjia & 121.42 & 28.62 & 1.3 \\
\hline 6 & Fuzhou & 119.28 & 26.08 & 84.0 \\
\hline 7 & Jianou & 118.32 & 27.05 & 154.9 \\
\hline 8 & Tunxi & 118.28 & 29.72 & 142.7 \\
\hline 9 & Hefei & 117.23 & 31.87 & 27.9 \\
\hline 10 & Gushi & 115.67 & 32.17 & 57.1 \\
\hline 11 & Wuhang & 114.13 & 30.62 & 23.1 \\
\hline 12 & Nanchang & 115.92 & 28.60 & 46.7 \\
\hline 13 & Ganzhou & 114.95 & 25.85 & 123.8 \\
\hline 14 & Shangtou & 116.68 & 23.40 & 2.9 \\
\hline 15 & Guangzhou & 113.33 & 23.17 & 41.0 \\
\hline 16 & Changling & 112.40 & 26.42 & 116.6 \\
\hline 17 & Changsha & 112.92 & 28.22 & 68.0 \\
\hline 18 & Yichang & 111.30 & 30.70 & 133.1 \\
\hline 19 & Nanyang & 112.58 & 33.03 & 129.2 \\
\hline 20 & Ankang & 109.03 & 32.72 & 290.8 \\
\hline 21 & Jishou & 109.73 & 28.32 & 208.4 \\
\hline 22 & Guiling & 110.30 & 25.32 & 164.4 \\
\hline 23 & Nanling & 108.22 & 22.63 & 121.6 \\
\hline 24 & Guiyang & 106.73 & 26.58 & 1223.8 \\
\hline 25 & Chongqing & 106.47 & 29.58 & 259.1 \\
\hline 26 & Mianyang & 104.75 & 31.45 & 486.3 \\
\hline 27 & Emeishan & 103.33 & 29.52 & 3047.4 \\
\hline 28 & Mengzhi & 103.38 & 23.38 & 1300.7 \\
\hline 29 & Kunming & 102.68 & 25.02 & 1892.4 \\
\hline 30 & Panzhihua & 101.72 & 26.58 & 1190.1 \\
\hline 31 & Lijiang & 100.22 & 26.87 & 2392.4 \\
\hline 32 & Tengchong & 98.50 & 25.02 & 1654.6 \\
\hline
\end{tabular}

applications of those models to other site where solar radiation data is not available. Therefore, exploring model developed at source site for estimating solar radiation at estimation site is of vital importance and significance, not only for China's subtropical zone but also for other regions. The main objectives of this study 
Table 2

RMSE $\left(\mathrm{MJ} \mathrm{m}^{-2}\right.$ ) of SVM models. ${ }^{\mathrm{a}}$

\begin{tabular}{|c|c|c|c|c|c|c|c|c|c|c|c|c|c|c|c|c|c|c|}
\hline Site ID $^{b}$ & 1 & 2 & 3 & 4 & 5 & 6 & 7 & 8 & 9 & 10 & 11 & 12 & 13 & 15 & 16 & 17 & \multicolumn{2}{|c|}{18} \\
\hline 1 & 1.96 & $2.16^{1}$ & $3.69^{2}$ & $4.3^{2}$ & $3.38^{2}$ & $3.97^{2}$ & $4.53^{2}$ & $5.45^{2}$ & $3.99^{2}$ & $3.59^{2}$ & $3.92^{2}$ & $3.8^{2}$ & $5.07^{2}$ & $5.16^{2}$ & $5.12^{2}$ & $4.58^{2}$ & \multicolumn{2}{|c|}{$4.57^{2}$} \\
\hline 2 & $2.27^{2}$ & 1.73 & $2.65^{2}$ & $3.17^{2}$ & $2.93^{2}$ & $3.1^{2}$ & $3.41^{2}$ & $4.38^{2}$ & $3^{2}$ & $3.24^{2}$ & $3.1^{2}$ & $3.08^{2}$ & $3.63^{2}$ & $4.36^{2}$ & $3.99^{2}$ & $3.48^{2}$ & 3.4 & \\
\hline 3 & $3.04^{2}$ & $2.47^{2}$ & 1.28 & $1.41^{1}$ & $3.09^{2}$ & $1.77^{2}$ & $2.35^{2}$ & $2.09^{2}$ & $1.35^{1}$ & $1.59^{2}$ & $1.6^{2}$ & $2.25^{2}$ & $1.63^{2}$ & $2.01^{2}$ & $1.81^{2}$ & $1.46^{1}$ & 1.8 & \\
\hline 4 & $3.46^{2}$ & $2.84^{2}$ & $1.57^{1}$ & 1.46 & $4.03^{2}$ & $1.72^{1}$ & $1.71^{1}$ & $2.2^{2}$ & $1.61^{1}$ & $2^{2}$ & $1.87^{2}$ & $2.32^{2}$ & $1.7^{1}$ & $2.08^{2}$ & $1.82^{2}$ & $1.67^{1}$ & 1.8 & \\
\hline 5 & $2.5^{2}$ & $1.91^{2}$ & $2.14^{2}$ & $2.41^{2}$ & 1.32 & $2.55^{2}$ & $2.81^{2}$ & $3.75^{2}$ & $2.31^{2}$ & $1.89^{2}$ & $2.3^{2}$ & $1.75^{2}$ & $2.67^{2}$ & $3.55^{2}$ & $3.33^{2}$ & $2.84^{2}$ & 3.1 & \\
\hline 6 & $3.65^{2}$ & $2.94^{2}$ & $1.8^{1}$ & $1.86^{1}$ & $4.52^{2}$ & 1.78 & $2.77^{2}$ & $2.28^{2}$ & $1.89^{1}$ & $2.1^{1}$ & $2.07^{1}$ & $2.53^{2}$ & $1.96^{1}$ & $2.39^{2}$ & $2.02^{1}$ & $1.85^{1}$ & 1.9 & \\
\hline 7 & $3.71^{2}$ & $3.48^{2}$ & $2.88^{1}$ & $2.78^{1}$ & $5.99^{2}$ & $2.88^{1}$ & 2.66 & $3^{1}$ & $2.96^{1}$ & $3.1^{1}$ & $3.25^{2}$ & $3.43^{2}$ & $3.59^{2}$ & $3.53^{2}$ & $2.78^{1}$ & $2.74^{1}$ & 3.0 & \\
\hline 8 & $3.27^{2}$ & $3.93^{2}$ & $1.71^{2}$ & $1.71^{2}$ & $5.7^{2}$ & $1.87^{2}$ & $1.57^{2}$ & 0.99 & $2.35^{2}$ & $2.26^{2}$ & $2.49^{2}$ & $3.03^{2}$ & $1.69^{2}$ & $3.28^{2}$ & $2.31^{2}$ & $1.6^{2}$ & 1.2 & \\
\hline 9 & $2.85^{2}$ & $2.18^{2}$ & $1.34^{2}$ & $1.31^{2}$ & $2.9^{2}$ & $1.73^{2}$ & $2.53^{2}$ & $2.2^{2}$ & 1.07 & $1.21^{1}$ & $1.32^{2}$ & $1.74^{2}$ & $1.33^{2}$ & $1.96^{2}$ & $1.82^{2}$ & $1.48^{2}$ & 1.8 & \\
\hline 10 & 2.872 & $2.32^{2}$ & $1.61^{2}$ & $1.86^{2}$ & $2.87^{2}$ & $2.18^{2}$ & $2.81^{2}$ & $2.63^{2}$ & $1.59^{2}$ & 1.29 & $1.76^{2}$ & $2.31^{2}$ & $1.83^{2}$ & $2.34^{2}$ & $2.35^{2}$ & $1.92^{2}$ & 2.3 & \\
\hline 11 & $3.21^{2}$ & $2.51^{2}$ & $1.42^{2}$ & $1.22^{1}$ & $2.82^{2}$ & $1.52^{2}$ & $1.49^{2}$ & $2.11^{2}$ & $1.27^{1}$ & $1.23^{1}$ & 1.11 & $1.56^{2}$ & $1.66^{2}$ & $2.3^{2}$ & $1.75^{2}$ & $1.35^{2}$ & 1.7 & \\
\hline 12 & $2.9^{2}$ & $2.2^{2}$ & $1.6^{2}$ & $1.94^{2}$ & $2.32^{2}$ & $1.78^{2}$ & $2.34^{2}$ & $3.15^{2}$ & $1.62^{2}$ & $1.41^{1}$ & $1.5^{2}$ & 1.2 & $1.87^{2}$ & $1.92^{2}$ & $2.39^{2}$ & $2.03^{2}$ & 2.3 & \\
\hline 13 & $3.56^{2}$ & $1.81^{2}$ & $1.7^{2}$ & $1.69^{2}$ & $5.46^{2}$ & $1.09^{2}$ & $1.71^{2}$ & $1.84^{2}$ & $1.16^{2}$ & $2.14^{2}$ & $1.64^{2}$ & $2.22^{2}$ & 0.87 & $1.13^{2}$ & $2.11^{2}$ & $1.55^{2}$ & 1.3 & \\
\hline 14 & $2.2^{2}$ & $1.82^{2}$ & $2.75^{2}$ & $3.61^{2}$ & $2.78^{2}$ & $3.27^{2}$ & $3.55^{2}$ & $4.5^{2}$ & $3.18^{2}$ & $2.56^{2}$ & $3.17^{2}$ & $2.46^{2}$ & $3.42^{2}$ & $4.92^{2}$ & $4.25^{2}$ & $3.43^{2}$ & 3.8 & \\
\hline 15 & $5.18^{2}$ & $4.08^{2}$ & $2.53^{2}$ & $2^{2}$ & $4.69^{2}$ & $2.31^{2}$ & $1.89^{2}$ & $2.04^{2}$ & $2.22^{2}$ & $2.99^{2}$ & $2.48^{2}$ & $3.19^{2}$ & $2.47^{2}$ & 1.31 & $2.02^{2}$ & $2.19^{2}$ & 1.9 & \\
\hline 16 & $5.07^{2}$ & $4.43^{2}$ & $3^{2}$ & $2.81^{1}$ & $5.34^{2}$ & $2.76^{1}$ & $2.7^{1}$ & $2.43^{1}$ & $2.99^{2}$ & $3.46^{2}$ & $3.22^{2}$ & $3.58^{2}$ & $2.81^{1}$ & $3.5^{2}$ & 2.41 & $2.57^{1}$ & 2.6 & \\
\hline 17 & $4.37^{2}$ & $3.62^{2}$ & $1.94^{1}$ & $1.9^{1}$ & $3.84^{2}$ & $1.89^{1}$ & $1.67^{1}$ & $1.75^{1}$ & $1.97^{2}$ & $2.47^{2}$ & $2.16^{2}$ & $2.5^{2}$ & $1.85^{1}$ & $2.65^{2}$ & $1.6^{-1}$ & 1.61 & 1.8 & \\
\hline 18 & $4^{2}$ & $3.68^{2}$ & $1.92^{2}$ & $1.78^{2}$ & $4.71^{2}$ & $1.66^{1}$ & $1.55^{1}$ & $1.39^{1}$ & $2.17^{2}$ & $2.58^{2}$ & $2.51^{2}$ & $2.91^{2}$ & $1.71^{2}$ & $2.99^{2}$ & $1.46^{1}$ & $1.52^{1}$ & 1.3 & \\
\hline 19 & $3.64^{2}$ & $3.23^{2}$ & $1.42^{2}$ & $1.86^{2}$ & $4.25^{2}$ & $1.7^{2}$ & $2.19^{2}$ & $1.49^{2}$ & $2.11^{2}$ & $1.86^{2}$ & $2.09^{2}$ & $3.52^{2}$ & $1.26^{2}$ & $2.97^{2}$ & $2.19^{2}$ & $1.24^{2}$ & 1.2 & \\
\hline 20 & $4.01^{2}$ & $4.04^{2}$ & $2.07^{2}$ & $2.33^{2}$ & $5.83^{2}$ & $2.17^{2}$ & $2.12^{2}$ & $1.71^{1}$ & $3.21^{2}$ & $2.66^{2}$ & $3.7^{2}$ & $4.77^{2}$ & $2^{2}$ & $4.51^{2}$ & $2.8^{2}$ & $2.16^{2}$ & 1.6 & \\
\hline 21 & $5.13^{2}$ & $4.74^{2}$ & $2.79^{2}$ & $2.59^{2}$ & $6.17^{2}$ & $2.5^{2}$ & $2.19^{2}$ & $1.45^{2}$ & $3.09^{2}$ & $3.45^{2}$ & $3.35^{2}$ & $3.81^{2}$ & $2.24^{2}$ & $3.59^{2}$ & $1.87^{2}$ & $2.08^{2}$ & 1.8 & \\
\hline 22 & $3.85^{2}$ & $3.15^{2}$ & $1.74^{2}$ & $1.66^{2}$ & $4.26^{2}$ & $1.66^{2}$ & $2.16^{2}$ & $1.98^{2}$ & $1.51^{1}$ & $1.95^{2}$ & $1.86^{2}$ & $2.32^{2}$ & $1.47^{1}$ & $1.72^{2}$ & $1.67^{2}$ & $1.58^{1}$ & 1.8 & \\
\hline 23 & $4.1^{2}$ & $3.39^{2}$ & $1.76^{2}$ & $1.7^{2}$ & $5.13^{2}$ & $1.69^{2}$ & $1.94^{2}$ & $1.43^{2}$ & $1.68^{2}$ & $2.19^{2}$ & $2.07^{2}$ & $3.09^{2}$ & $1.37^{2}$ & $1.91^{2}$ & $1.24^{1}$ & $1.25^{1}$ & 1.6 & \\
\hline 24 & $4.39^{2}$ & $3.73^{2}$ & $1.84^{2}$ & $1.87^{2}$ & $3.44^{2}$ & $1.75^{2}$ & $1.37^{2}$ & $2^{2}$ & $1.79^{2}$ & $2.04^{2}$ & $1.82^{2}$ & $2.48^{2}$ & $1.53^{2}$ & $2.56^{2}$ & $1.68^{2}$ & $1.38^{2}$ & 1.6 & \\
\hline 25 & $5.31^{2}$ & $4.51^{2}$ & $2.61^{2}$ & $2.32^{2}$ & $5.23^{2}$ & $2.41^{2}$ & $2.07^{2}$ & $1.41^{2}$ & $2.61^{2}$ & $3.23^{2}$ & $2.99^{2}$ & $3.51^{2}$ & $2.16^{2}$ & $2.31^{2}$ & $1.84^{2}$ & $2.01^{2}$ & 1.7 & \\
\hline 26 & $4.36^{2}$ & $3.88^{2}$ & $2.01^{2}$ & $1.82^{2}$ & $4.67^{2}$ & $1.87^{2}$ & $1.52^{2}$ & $1.33^{1}$ & $2.23^{2}$ & $2.5^{2}$ & $2.33^{2}$ & $3^{2}$ & $1.65^{2}$ & $2.77^{2}$ & $1.37^{2}$ & $1.56^{2}$ & 1.2 & \\
\hline 27 & $2.96^{2}$ & $1.26^{1}$ & $3.14^{2}$ & $2.89^{2}$ & $3.33^{2}$ & $4.07^{2}$ & $4.23^{2}$ & $4.41^{2}$ & $3.35^{2}$ & $5.31^{2}$ & $3.38^{2}$ & $8^{2}$ & $5.32^{2}$ & $2.94^{2}$ & $5.95^{2}$ & $4.56^{2}$ & $5^{2}$ & \\
\hline 28 & $2.55^{2}$ & $2.4^{1}$ & $2.98^{2}$ & $3.06^{2}$ & $3.88^{2}$ & $3.29^{2}$ & $4.02^{2}$ & $4.36^{2}$ & $2.69^{2}$ & $2.6^{2}$ & $2.55^{2}$ & $2.55^{2}$ & $3.42^{2}$ & $2.91^{2}$ & $3.82^{2}$ & $3.5^{2}$ & 3.8 & \\
\hline 29 & $2.41^{2}$ & $2^{2}$ & $2.4^{2}$ & $2.67^{2}$ & $3.18^{2}$ & $2.65^{2}$ & $3.14^{2}$ & $4.2^{2}$ & $1.94^{2}$ & $2.13^{2}$ & $1.84^{2}$ & $2^{2}$ & $3.68^{2}$ & $3.33^{2}$ & $3.31^{2}$ & $2.77^{2}$ & 3.7 & \\
\hline 30 & $2.44^{2}$ & $3.51^{2}$ & $2.09^{1}$ & $2.11^{1}$ & $10.81^{2}$ & $2.56^{2}$ & $2.19^{1}$ & $2.73^{2}$ & $3.23^{2}$ & $2.9^{2}$ & $4.15^{2}$ & $8.87^{2}$ & $2.65^{2}$ & $5.77^{2}$ & $3.4^{2}$ & $2.14^{1}$ & 2.1 & \\
\hline 31 & $1.85^{2}$ & $1.69^{2}$ & $3.36^{2}$ & $2.79^{2}$ & $3.45^{2}$ & $2.95^{2}$ & $3.73^{2}$ & $4.75^{2}$ & $2.16^{2}$ & $3.33^{2}$ & $2.15^{2}$ & $2.2^{2}$ & $3.67^{2}$ & $2.28^{2}$ & $3.4^{2}$ & $3.16^{2}$ & 4.2 & \\
\hline 32 & $2.53^{2}$ & $2.28^{2}$ & $1.88^{2}$ & $1.95^{2}$ & $3.56^{2}$ & $2.29^{2}$ & $5.37^{2}$ & $3.84^{2}$ & $2.42^{2}$ & $2.34^{2}$ & $2.46^{2}$ & $3.68^{2}$ & $3.14^{2}$ & $4.09^{2}$ & $3.41^{2}$ & $2.83^{2}$ & 3.1 & \\
\hline Average & 3.42 & 2.94 & 2.17 & 2.21 & 4.25 & 2.29 & 2.57 & 2.66 & 2.27 & 2.49 & 2.44 & 3.11 & 2.42 & 2.97 & 2.60 & 2.25 & 2.4 & \\
\hline & & & & & & & & & & & & & & & & & $\mathrm{mb}$ & \\
\hline Site ID & 19 & 20 & 21 & 22 & 23 & 24 & 25 & 26 & 27 & 28 & & 30 & & 32 & & -1 & 1 & 2 \\
\hline 1 & $3.97^{2}$ & $4.33^{2}$ & $6.24^{2}$ & $5.48^{2}$ & $5.79^{2}$ & $4.43^{2}$ & $5.9^{2}$ & $5.02^{2}$ & $2.55^{2}$ & $2.97^{2}$ & $2.38^{2}$ & $2.77^{2}$ & $2.31^{1}$ & $5.75^{2}$ & 4.12 & 0 & 2 & 30 \\
\hline 2 & $4.17^{2}$ & $3.56^{2}$ & $5.29^{2}$ & $4.14^{2}$ & $4.35^{2}$ & $3.57^{2}$ & $4.83^{2}$ & $3.76^{2}$ & $8.82^{2}$ & $3^{2}$ & $2.49^{2}$ & $2.29^{2}$ & $1.97^{1}$ & $4.8^{2}$ & 3.58 & 0 & 1 & 31 \\
\hline 3 & $1.8^{2}$ & $1.94^{2}$ & $3.09^{2}$ & $2.22^{2}$ & $2.02^{2}$ & $2.3^{2}$ & $2.9^{2}$ & $2.04^{2}$ & $8.98^{2}$ & $5.86^{2}$ & $3.48^{2}$ & $1.61^{2}$ & $2.73^{2}$ & $3.22^{2}$ & 2.57 & 0 & 3 & 29 \\
\hline 4 & $1.7^{1}$ & $1.82^{2}$ & $2.76^{2}$ & $1.95^{2}$ & $2.13^{2}$ & $3^{2}$ & $2.61^{2}$ & $1.91^{2}$ & $8.58^{2}$ & $4.05^{2}$ & $6.16^{2}$ & $2.06^{2}$ & $2.7^{2}$ & $4.85^{2}$ & 2.74 & 0 & 7 & 25 \\
\hline 5 & $3.04^{2}$ & $3.09^{2}$ & $4.66^{2}$ & $2.9^{2}$ & $3.55^{2}$ & $2.65^{2}$ & $4.57^{2}$ & $3.32^{2}$ & $10.14^{2}$ & $3.42^{2}$ & $3.04^{2}$ & $2.47^{2}$ & $3.55^{2}$ & $3.83^{2}$ & 3.11 & 0 & 0 & 32 \\
\hline 6 & $1.81^{1}$ & $1.9^{1}$ & $2.8^{2}$ & $2.03^{1}$ & $2.14^{1}$ & $3.31^{2}$ & $2.87^{2}$ & $2.06^{1}$ & $3.39^{2}$ & $3.93^{2}$ & $8.14^{2}$ & $2.63^{2}$ & $2.99^{2}$ & $3.7^{2}$ & 2.78 & 0 & 14 & 18 \\
\hline 7 & $2.97^{1}$ & $3.15^{1}$ & $3.43^{2}$ & $3.48^{2}$ & $2.86^{1}$ & $3.67^{2}$ & $3.42^{2}$ & $3.1^{1}$ & $3.9^{2}$ & $5.65^{2}$ & $7.97^{2}$ & $3.28^{2}$ & $3.71^{2}$ & $4.16^{2}$ & 3.63 & 0 & 13 & 19 \\
\hline 8 & $1.56^{2}$ & $1.21^{2}$ & $1.44^{2}$ & $3.38^{2}$ & $1.57^{2}$ & $2.6^{2}$ & $1.36^{2}$ & $1.22^{2}$ & $7.66^{2}$ & $4.82^{2}$ & $8.72^{2}$ & $2.07^{2}$ & $4.53^{2}$ & $4.79^{2}$ & 3.00 & 0 & 0 & 32 \\
\hline 9 & $1.7^{2}$ & $1.97^{2}$ & $3.15^{2}$ & $1.41^{2}$ & $1.78^{2}$ & $2.32^{2}$ & $3.06^{2}$ & $2.1^{2}$ & $8.72^{2}$ & $3.66^{2}$ & $3.14^{2}$ & $1.5^{2}$ & $2.14^{2}$ & $3.15^{2}$ & 2.34 & 0 & 1 & 31 \\
\hline 10 & $1.97^{2}$ & $2.42^{2}$ & $3.69^{2}$ & $2.39^{2}$ & $2.52^{2}$ & $2.23^{2}$ & $3.51^{2}$ & $2.54^{2}$ & $7.4^{2}$ & $3.64^{2}$ & $2.97^{2}$ & $1.95^{2}$ & $2.39^{2}$ & $3.18^{2}$ & 2.63 & 0 & 0 & 32 \\
\hline 11 & $1.48^{2}$ & $1.7^{2}$ & $2.91^{2}$ & $1.37^{2}$ & $1.69^{2}$ & $2.39^{2}$ & $3.07^{2}$ & $1.86^{2}$ & $2.38^{2}$ & $3.71^{2}$ & $5.64^{2}$ & $3.39^{2}$ & $3.09^{2}$ & $3.66^{2}$ & 2.27 & 0 & 3 & 29 \\
\hline 12 & $1.74^{2}$ & $2.15^{2}$ & $3.68^{2}$ & $2.21^{2}$ & $2.52^{2}$ & $2.36^{2}$ & $3.77^{2}$ & $2.42^{2}$ & $3.1^{2}$ & $3.48^{2}$ & $2.77^{2}$ & $3.12^{2}$ & $2.68^{2}$ & $4.48^{2}$ & 2.45 & 0 & 1 & 31 \\
\hline 13 & $1.01^{1}$ & $1.37^{2}$ & $2.27^{2}$ & $1.51^{2}$ & $1.37^{2}$ & $3.44^{2}$ & $2.28^{2}$ & $1.42^{2}$ & $2.31^{2}$ & $4.88^{2}$ & $3.77^{2}$ & $0.54^{-1}$ & $3.13^{2}$ & $3.72^{2}$ & 2.25 & 1 & 1 & 30 \\
\hline 14 & $2.87^{2}$ & $3.77^{2}$ & $5.69^{2}$ & $4.12^{2}$ & $4.23^{2}$ & $2.83^{2}$ & $5.75^{2}$ & $3.87^{2}$ & $10.67^{2}$ & $3.47^{2}$ & $4.09^{2}$ & $3.12^{2}$ & $1.99^{2}$ & $2.12^{2}$ & 3.61 & 0 & 0 & 32 \\
\hline 15 & $2.78^{2}$ & $1.96^{2}$ & $2.11^{2}$ & $1.75^{2}$ & $1.98^{2}$ & $4.07^{2}$ & $2.13^{2}$ & $2.01^{2}$ & $2.74^{2}$ & $4.82^{2}$ & $6.57^{2}$ & $3.73^{2}$ & $4.71^{2}$ & $16.42^{2}$ & 3.40 & 0 & 0 & 32 \\
\hline 16 & $2.76^{1}$ & $2.68^{1}$ & $2.56^{1}$ & $2.99^{2}$ & $2.49^{1}$ & $3.7^{2}$ & $2.69^{1}$ & $2.5^{1}$ & $4.99^{2}$ & $5.56^{2}$ & $5.2^{2}$ & $4.75^{2}$ & $4.18^{2}$ & $4.99^{2}$ & 3.56 & 0 & 13 & 19 \\
\hline 17 & $1.86^{1}$ & $1.76^{1}$ & $2.29^{2}$ & $2.02^{2}$ & $1.7^{1}$ & $2.97^{2}$ & $2.39^{2}$ & $1.71^{1}$ & $4.02^{2}$ & $5.78^{2}$ & $4.08^{2}$ & $4.03^{2}$ & $4.5^{2}$ & $4.39^{2}$ & 2.78 & 1 & 11 & 20 \\
\hline 18 & $1.57^{1}$ & $1.48^{1}$ & $1.76^{2}$ & $2.79^{2}$ & $1.64^{1}$ & $2.99^{2}$ & $1.7^{2}$ & $1.35^{-1}$ & $3.07^{2}$ & $4.44^{2}$ & $4.63^{2}$ & $3.34^{2}$ & $3.08^{2}$ & $3.32^{2}$ & 2.63 & 1 & 8 & 23 \\
\hline 19 & 0.84 & $1.36^{2}$ & $2.31^{2}$ & $3.75^{2}$ & $1.97^{2}$ & $2.57^{2}$ & $2.2^{2}$ & $1.38^{2}$ & $2.54^{2}$ & $5.76^{2}$ & $4.24^{2}$ & $1.59^{2}$ & $3.29^{2}$ & $2.75^{2}$ & 2.55 & 0 & 0 & 32 \\
\hline 20 & $1.75^{1}$ & 1.47 & $1.88^{2}$ & $2.51^{2}$ & $2.29^{2}$ & $3.18^{2}$ & $1.81^{2}$ & $1.61^{1}$ & $3.3^{2}$ & $4.15^{2}$ & $7.26^{2}$ & $3.48^{2}$ & $4.08^{2}$ & $4.02^{2}$ & 3.25 & 0 & 4 & 28 \\
\hline 21 & $2.22^{2}$ & $1.84^{2}$ & 1.04 & $3.5^{2}$ & $1.86^{2}$ & $3.2^{2}$ & $1.24^{1}$ & $1.59^{2}$ & $3.93^{2}$ & $5.61^{2}$ & $5.54^{2}$ & $4.57^{2}$ & $4.68^{2}$ & $4.61^{2}$ & 3.33 & 0 & 1 & 31 \\
\hline 22 & $1.78^{2}$ & $1.84^{2}$ & $2.55^{2}$ & 1.33 & $1.63^{2}$ & $2.28^{2}$ & $2.58^{2}$ & $1.86^{2}$ & $3.36^{2}$ & $4.56^{2}$ & $3.66^{2}$ & $3.49^{2}$ & $3.12^{2}$ & $3.9^{2}$ & 2.47 & 0 & 3 & 29 \\
\hline 23 & $1.67^{2}$ & $1.62^{2}$ & $2.02^{2}$ & $1.98^{2}$ & 1.13 & $2.57^{2}$ & $2.27^{2}$ & $1.51^{2}$ & $3.34^{2}$ & $5.73^{2}$ & $4.23^{2}$ & $2.94^{2}$ & $2.84^{2}$ & $17.35^{2}$ & 2.96 & 0 & 2 & 30 \\
\hline 24 & $1.6^{2}$ & $1.56^{2}$ & $2.46^{2}$ & $1.83^{2}$ & $1.58^{2}$ & 1.13 & $2.37^{2}$ & $1.74^{2}$ & $3.71^{2}$ & $4.62^{2}$ & $3.64^{2}$ & $1.65^{2}$ & $3.83^{2}$ & $3.74^{2}$ & 2.45 & 0 & 0 & 32 \\
\hline 25 & $2.35^{2}$ & $2.25^{2}$ & $0.95^{1}$ & $2.48^{2}$ & $1.82^{2}$ & $3.4^{2}$ & 0.85 & $1.48^{2}$ & $3.41^{2}$ & $6.03^{2}$ & $5.3^{2}$ & $4.82^{2}$ & $3.57^{2}$ & $4.78^{2}$ & 3.08 & 0 & 1 & 31 \\
\hline 26 & $1.6^{2}$ & $1.24^{1}$ & $1.52^{2}$ & $2.37^{2}$ & $1.51^{2}$ & $2.46^{2}$ & $1.39^{2}$ & 1.16 & $3.42^{2}$ & $4.99^{2}$ & $4.52^{2}$ & $4.18^{2}$ & $3.89^{2}$ & $3.85^{2}$ & 2.65 & 0 & 3 & 29 \\
\hline 27 & $3.87^{2}$ & $3.76^{2}$ & $8.76^{2}$ & $7.92^{2}$ & $7.58^{2}$ & $6^{2}$ & $8.1^{2}$ & $5.63^{2}$ & 1.15 & $2.03^{2}$ & $1.32^{1}$ & $4.61^{2}$ & $1.96^{2}$ & $4.28^{2}$ & 4.47 & 0 & 2 & 30 \\
\hline 28 & $3.53^{2}$ & $4.23^{2}$ & $5.15^{2}$ & $2.76^{2}$ & $3.68^{2}$ & $3.19^{2}$ & $4.75^{2}$ & $4.1^{2}$ & $2.63^{2}$ & 2.11 & $2.69^{2}$ & $2.68^{2}$ & $2.8^{2}$ & $2.5^{2}$ & 3.29 & 0 & 1 & 31 \\
\hline 29 & $3.23^{2}$ & $4.35^{2}$ & $5.21^{2}$ & $2.08^{2}$ & $3.83^{2}$ & $3.29^{2}$ & $4.22^{2}$ & $4.05^{2}$ & $2.41^{2}$ & $1.94^{2}$ & 1.33 & $2.46^{2}$ & $1.45^{1}$ & $2.11^{2}$ & 2.91 & 0 & 1 & 31 \\
\hline 30 & $2.16^{1}$ & $2.64^{2}$ & $2.58^{2}$ & $6.08^{2}$ & $2.14^{1}$ & $3.28^{2}$ & $2.68^{2}$ & $2.43^{2}$ & $3^{2}$ & $5.1^{2}$ & $5.19^{2}$ & 1.97 & $2.75^{2}$ & $2.86^{2}$ & 3.70 & 0 & 7 & 25 \\
\hline 31 & $3.96^{2}$ & $5.17^{2}$ & $6.17^{2}$ & $1.71^{2}$ & $5.26^{2}$ & $4.28^{2}$ & $4.49^{2}$ & $4.68^{2}$ & $2.14^{2}$ & $2.02^{2}$ & $1.44^{1}$ & $3.05^{2}$ & 1.41 & $2.48^{2}$ & 3.27 & 0 & 1 & 31 \\
\hline 32 & $2.74^{2}$ & $3.75^{2}$ & $4.91^{2}$ & $3.77^{2}$ & $4.25^{2}$ & $3.08^{2}$ & $4.34^{2}$ & $3.43^{2}$ & $3.44^{2}$ & $2.41^{2}$ & $0.94^{-1}$ & $2.34^{2}$ & $1.21^{-1}$ & 1.51 & 3.13 & 2 & 0 & 30 \\
\hline Average & 2.31 & 2.48 & 3.35 & 2.88 & 2.71 & 3.09 & 3.19 & 2.53 & 4.54 & 4.19 & 4.27 & 2.89 & 3.04 & 4.54 & - & - & - & - \\
\hline
\end{tabular}

a Superscript -1 indicates RMSE of SVM developed at source site (first row) is lower than that (diagonal) driven by estimation (first column) site's own data, 1 indicates the difference of RMSE between source site and estimation sites less than $20 \%$, and 2 indicates other source sites.

${ }^{b}$ Site ID in first row represent for the sites (sources site) that provide data constructing SVM model for solar radiation estimation at the site (estimation site) indicated by site ID in the first column. 
Table 3

MAPE of SVM models.

\begin{tabular}{|c|c|c|c|c|c|c|c|c|c|c|c|c|c|c|c|c|c|}
\hline Site ID ${ }^{a}$ & 1 & 2 & 3 & 4 & & 5 & 6 & 7 & 8 & 9 & 10 & 11 & 12 & 13 & 14 & 15 & 16 \\
\hline 1 & $9.9 \%$ & $11.5 \%$ & $22.4^{\circ}$ & 26.7 & & $.5 \%$ & $7 \%$ & $30.7 \%$ & $37.7 \%$ & $25.6 \%$ & $22.8 \%$ & $25.4 \%$ & $24.3 \%$ & $34.8 \%$ & $15.7 \%$ & $28.9 \%$ & $35.9 \%$ \\
\hline 2 & $13.6 \%$ & $10.2 \%$ & $18.3^{\circ}$ & 21.5 & & $7.1 \%$ & $9 \%$ & $25.3 \%$ & $32.9 \%$ & $20.6 \%$ & $20.8 \%$ & $21.7 \%$ & $19.9 \%$ & $26.7 \%$ & $16.7 \%$ & $27.9 \%$ & $30.3 \%$ \\
\hline 3 & $24.9 \%$ & $19.5 \%$ & $8.0 \%$ & $9.8^{\circ}$ & & $3.9 \%$ & $.0 \%$ & $15.5 \%$ & $14.2 \%$ & $8.6 \%$ & $9.8 \%$ & $10.3 \%$ & $14.8 \%$ & $11.1 \%$ & $33.9 \%$ & $13.2 \%$ & $12.6 \%$ \\
\hline 4 & $28.5 \%$ & $22.4 \%$ & $10.5^{\circ}$ & $9.3^{\circ}$ & & $.9 \%$ & $6 \%$ & $11.6 \%$ & $16.1 \%$ & $10.8 \%$ & $12.8 \%$ & $12.0 \%$ & $14.9 \%$ & $11.6 \%$ & $44.7 \%$ & $15.1 \%$ & $13.2 \%$ \\
\hline 5 & $13.8 \%$ & $12.3 \%$ & $13.2^{\circ}$ & 15.3 & & $.6 \%$ & $5 \%$ & $19.5 \%$ & $26.3 \%$ & $13.2 \%$ & $12.7 \%$ & $14.1 \%$ & $10.5 \%$ & $17.3 \%$ & $15.2 \%$ & $21.3 \%$ & $22.2 \%$ \\
\hline 6 & $28.7 \%$ & $22.0 \%$ & $10.7^{\circ}$ & 10.6 & & $.1 \%$ & $8 \%$ & $16.7 \%$ & $14.9 \%$ & $11.3 \%$ & $13.0 \%$ & $12.5 \%$ & $15.9 \%$ & $12.0 \%$ & $32.6 \%$ & $14.7 \%$ & $12.6 \%$ \\
\hline 7 & $26.9 \%$ & $25.1 \%$ & $14.9^{\circ}$ & 14.4 & & $4.4 \%$ & $.1 \%$ & $11.7 \%$ & $12.9 \%$ & $17.7 \%$ & $18.9 \%$ & $19.9 \%$ & $22.3 \%$ & $16.3 \%$ & $36.1 \%$ & $23.6 \%$ & $12.8 \%$ \\
\hline 8 & $22.7 \%$ & $34.1 \%$ & $9.5 \%$ & 11.1 & & $5.2 \%$ & $5 \%$ & $8.9 \%$ & $6.2 \%$ & $18.0 \%$ & $13.2 \%$ & $18.5 \%$ & $23.5 \%$ & $11.9 \%$ & $58.0 \%$ & $26.6 \%$ & $15.4 \%$ \\
\hline 9 & $24.2 \%$ & $18.1 \%$ & $7.0 \%$ & $8.3^{\circ}$ & & $.0 \%$ & $3 \%$ & $15.9 \%$ & $15.7 \%$ & $6.3 \%$ & $7.9 \%$ & $8.9 \%$ & $12.4 \%$ & $9.2 \%$ & $30.2 \%$ & $13.7 \%$ & $13.3 \%$ \\
\hline 10 & $24.0 \%$ & $18.4 \%$ & $9.8 \%$ & 11.8 & & $.5 \%$ & $5 \%$ & $18.2 \%$ & $18.2 \%$ & $9.8 \%$ & $7.1 \%$ & $12.0 \%$ & $16.0 \%$ & $12.7 \%$ & $30.4 \%$ & $15.7 \%$ & $16.6 \%$ \\
\hline 11 & $26.7 \%$ & $21.5 \%$ & $7.0 \%$ & $8.4^{\circ}$ & & $.6 \%$ & $3 \%$ & $11.2 \%$ & $16.1 \%$ & $8.8 \%$ & $7.9 \%$ & $7.2 \%$ & $11.0 \%$ & $11.8 \%$ & $29.3 \%$ & $20.0 \%$ & $13.8 \%$ \\
\hline 12 & $24.2 \%$ & $17.8 \%$ & $11.0^{\circ}$ & 12.6 & & $1.6 \%$ & $9 \%$ & $16.3 \%$ & $22.9 \%$ & $11.0 \%$ & $10.2 \%$ & $11.0 \%$ & $7.8 \%$ & $14.6 \%$ & $28.0 \%$ & $21.3 \%$ & $18.3 \%$ \\
\hline 13 & $30.7 \%$ & $13.4 \%$ & $12.7^{\circ}$ & 11.8 & & $7.3 \%$ & $2 \%$ & $10.9 \%$ & $13.2 \%$ & $8.2 \%$ & $15.7 \%$ & $10.2 \%$ & $15.0 \%$ & $5.5 \%$ & $42.8 \%$ & $7.1 \%$ & $16.5 \%$ \\
\hline 14 & $13.6 \%$ & $11.0 \%$ & $16.5^{\circ}$ & 21.6 & & $.9 \%$ & $.0 \%$ & $22.6 \%$ & $30.0 \%$ & $19.8 \%$ & $15.7 \%$ & $20.0 \%$ & $14.6 \%$ & $22.4 \%$ & $7.9 \%$ & $31.3 \%$ & $28.4 \%$ \\
\hline 15 & $43.8 \%$ & $34.7 \%$ & $20.2^{\circ}$ & 16.4 & & $1.7 \%$ & $6 \%$ & $13.3 \%$ & $15.8 \%$ & $17.2 \%$ & $22.3 \%$ & $19.6 \%$ & $24.9 \%$ & $17.4 \%$ & $46.0 \%$ & $9.2 \%$ & $15.7 \%$ \\
\hline 16 & $58.7 \%$ & $50.5 \%$ & $27.0^{\circ}$ & 27.2 & & $.4 \%$ & $6 \%$ & $22.3 \%$ & $20.4 \%$ & $29.7 \%$ & $34.7 \%$ & $31.8 \%$ & $35.4 \%$ & $23.1 \%$ & $69.2 \%$ & $42.2 \%$ & $19.1 \%$ \\
\hline 17 & $45.8 \%$ & $37.4 \%$ & $16.5^{\circ}$ & 16.6 & & $.5 \%$ & $.1 \%$ & $2.7 \%$ & $12.9 \%$ & $17.4 \%$ & $21.5 \%$ & $18.5 \%$ & $21.6 \%$ & $13.7 \%$ & $59.2 \%$ & $6 \%$ & $11.7 \%$ \\
\hline 18 & $43.7 \%$ & $38.8 \%$ & $18.4^{\circ}$ & 17.5 & & $7.3 \%$ & $3 \%$ & $4.6 \%$ & $14.7 \%$ & $19.7 \%$ & $23.3 \%$ & $21.5 \%$ & $25.6 \%$ & $16.4 \%$ & $67.0 \%$ & $33.4 \%$ & $15.4 \%$ \\
\hline 19 & $33.0 \%$ & $28.4 \%$ & $9.8 \%$ & 14.8 & & $3.3 \%$ & $.1 \%$ & $14.9 \%$ & $10.1 \%$ & $13.8 \%$ & $11.5 \%$ & $13.2 \%$ & $23.7 \%$ & $8.3 \%$ & $45.8 \%$ & 18. & $15.6 \%$ \\
\hline 20 & $39.4 \%$ & $36.6 \%$ & $15.7^{\circ}$ & 18.9 & & $.9 \%$ & $1 \%$ & $2.8 \%$ & $10.7 \%$ & $21.8 \%$ & $20.3 \%$ & $23.4 \%$ & $28.4 \%$ & $13.2 \%$ & $73.3 \%$ & $31.0 \%$ & $18.7 \%$ \\
\hline 21 & $60.1 \%$ & $54.0 \%$ & $22.3^{\circ}$ & 27.5 & & $.4 \%$ & $8 \%$ & $1.2 \%$ & $12.9 \%$ & $31.8 \%$ & $35.8 \%$ & $32.1 \%$ & $38.0 \%$ & $21.3 \%$ & $82.7 \%$ & $40.7 \%$ & $16.8 \%$ \\
\hline 22 & $34.5 \%$ & $27.7 \%$ & $14.8^{\circ}$ & 13.0 & & $7.4 \%$ & $6 \%$ & $15.4 \%$ & $14.5 \%$ & $12.4 \%$ & $16.2 \%$ & $15.4 \%$ & $18.4 \%$ & $11.0 \%$ & $38.7 \%$ & & $12.2 \%$ \\
\hline 23 & $37.4 \%$ & $31.1 \%$ & $15.2^{\circ}$ & 14.5 & & $5 \%$ & $4 \%$ & $2.6 \%$ & $9.7 \%$ & $14.0 \%$ & $19.0 \%$ & $17.2 \%$ & $23.6 \%$ & $10.1 \%$ & $48.3 \%$ & $13.9 \%$ & $9.1 \%$ \\
\hline 24 & $49.3 \%$ & $41.2 \%$ & $19.5^{\circ}$ & 18.9 & & $2.8 \%$ & $.0 \%$ & $11.8 \%$ & $14.4 \%$ & $18.6 \%$ & $21.2 \%$ & $17.9 \%$ & $22.5 \%$ & $14.4 \%$ & $62.2 \%$ & $29.1 \%$ & $12.6 \%$ \\
\hline 25 & $69.1 \%$ & $57.9 \%$ & $31.1^{\circ}$ & 23.5 & & $.4 \%$ & $2 \%$ & $\%$ & & $24.3 \%$ & & $31.7 \%$ & & $17.1 \%$ & 63. & & $16.0 \%$ \\
\hline 26 & $48.3 \%$ & $41.2 \%$ & $18.3^{\circ}$ & 15.9 & & $.5 \%$ & $6 \%$ & $11.2 \%$ & $10.9 \%$ & $18.4 \%$ & $21.6 \%$ & $19.1 \%$ & $25.5 \%$ & $12.9 \%$ & $58.8 \%$ & $29.7 \%$ & $11.3 \%$ \\
\hline 27 & $17.8 \%$ & $7.7 \%$ & $21.8^{\circ}$ & 17.6 & & $.6 \%$ & $4 \%$ & $29.8 \%$ & $27.9 \%$ & $23.7 \%$ & $35.2 \%$ & $24.1 \%$ & $51.8 \%$ & $38.4 \%$ & $37.0 \%$ & $18.9 \%$ & $44.2 \%$ \\
\hline 28 & $12.7 \%$ & $11.8 \%$ & $14.6^{\circ}$ & 15.3 & & $7.4 \%$ & $2 \%$ & $2.6 \%$ & $25.0 \%$ & $13.3 \%$ & $12.6 \%$ & $12.5 \%$ & $11.9 \%$ & $17.7 \%$ & $20.8 \%$ & $14.7 \%$ & $21.2 \%$ \\
\hline 29 & $12.8 \%$ & $10.4 \%$ & $13.0^{\circ}$ & 13.4 & & $.4 \%$ & $9 \%$ & $18.2 \%$ & $25.8 \%$ & $10.5 \%$ & $11.2 \%$ & $10.0 \%$ & $9.6 \%$ & $19.2 \%$ & $20.3 \%$ & $18.9 \%$ & $19.7 \%$ \\
\hline 30 & $13.4 \%$ & $21.2 \%$ & $10.5^{\circ}$ & 11.1 & & $7.1 \%$ & $8 \%$ & $11.0 \%$ & $15.0 \%$ & $17.4 \%$ & $15.6 \%$ & $22.4 \%$ & $38.0 \%$ & $13.3 \%$ & $58.9 \%$ & $33.1 \%$ & $19.1 \%$ \\
\hline 31 & $9.2 \%$ & $8.0 \%$ & $16.7^{\circ}$ & 12.9 & & $.2 \%$ & $2 \%$ & $19.8 \%$ & $26.2 \%$ & $10.5 \%$ & $16.4 \%$ & $10.3 \%$ & $10.7 \%$ & $19.1 \%$ & $28.4 \%$ & $11.3 \%$ & $17.5 \%$ \\
\hline 32 & $15.9 \%$ & $12.2 \%$ & $10.4^{\circ}$ & 10.7 & & $.5 \%$ & $9 \%$ & $4.9 \%$ & $23.2 \%$ & $13.9 \%$ & $14.7 \%$ & $13.7 \%$ & $17.1 \%$ & $18.3 \%$ & $32.1 \%$ & $23.9 \%$ & $20.3 \%$ \\
\hline Average & $29.9 \%$ & $25.3 \%$ & $15.2^{\circ}$ & 15.6 & & $7.5 \%$ & $4 \%$ & $17.3 \%$ & $18.2 \%$ & $16.2 \%$ & $17.9 \%$ & $17.4 \%$ & $21.5 \%$ & $16.3 \%$ & $41.7 \%$ & $22.5 \%$ & $18.1 \%$ \\
\hline Site ID ${ }^{\text {a }}$ & 17 & 18 & 19 & 20 & 21 & 22 & 23 & 24 & 25 & 26 & 27 & 28 & 29 & 30 & 31 & 32 & Average \\
\hline 1 & $32.0 \%$ & $30.5 \%$ & $26.8 \%$ & $29.7 \%$ & $43.9 \%$ & $37.9 \%$ & $42.1 \%$ & $31.9 \%$ & $40.0 \%$ & $34.5 \%$ & $12.4 \%$ & $17.0 \%$ & $12.7 \%$ & $16.9 \%$ & $12.6 \%$ & $39.9 \%$ & $26.8 \%$ \\
\hline 2 & $26.7 \%$ & $24.3 \%$ & $27.5 \%$ & $26.1 \%$ & $40.1 \%$ & $29.8 \%$ & $34.1 \%$ & $27.9 \%$ & $35.9 \%$ & $27.7 \%$ & $40.7 \%$ & $19.8 \%$ & $14.5 \%$ & $14.4 \%$ & $13.7 \%$ & $36.9 \%$ & $24.6 \%$ \\
\hline 3 & $9.7 \%$ & $12.1 \%$ & $11.2 \%$ & $12.9 \%$ & $24.2 \%$ & $15.7 \%$ & $15.6 \%$ & $16.9 \%$ & $19.6 \%$ & $14.5 \%$ & $45.6 \%$ & $37.8 \%$ & $24.1 \%$ & $11.3 \%$ & $22.1 \%$ & $24.5 \%$ & $17.4 \%$ \\
\hline 4 & $11.8 \%$ & $11.6 \%$ & $11.4 \%$ & $12.5 \%$ & $22.0 \%$ & $12.7 \%$ & $17.2 \%$ & $19.7 \%$ & $19.2 \%$ & $13.1 \%$ & $44.2 \%$ & $31.1 \%$ & $30.6 \%$ & $12.6 \%$ & $21.6 \%$ & $42.4 \%$ & $18.9 \%$ \\
\hline 5 & $19.1 \%$ & $18.7 \%$ & $19.4 \%$ & $19.8 \%$ & $33.3 \%$ & $17.6 \%$ & $26.1 \%$ & $19.3 \%$ & $29.2 \%$ & $22.0 \%$ & $46.1 \%$ & $19.9 \%$ & $17.9 \%$ & $13.5 \%$ & $19.2 \%$ & $23.2 \%$ & $19.2 \%$ \\
\hline 6 & $11.3 \%$ & $11.4 \%$ & $10.9 \%$ & $11.4 \%$ & $19.5 \%$ & $12.2 \%$ & $13.7 \%$ & $18.7 \%$ & $18.1 \%$ & $12.0 \%$ & $25.7 \%$ & $30.0 \%$ & $40.9 \%$ & $20.3 \%$ & $22.3 \%$ & $27.2 \%$ & $17.8 \%$ \\
\hline 7 & $12.6 \%$ & $13.4 \%$ & $13.4 \%$ & $14.0 \%$ & $16.1 \%$ & $21.4 \%$ & $13.3 \%$ & $18.0 \%$ & $14.5 \%$ & $13.2 \%$ & $28.1 \%$ & $35.7 \%$ & $41.7 \%$ & $22.0 \%$ & $24.6 \%$ & $28.0 \%$ & $20.4 \%$ \\
\hline 8 & $10.2 \%$ & $9.0 \%$ & $10.9 \%$ & $8.0 \%$ & $11.1 \%$ & $23.2 \%$ & $11.0 \%$ & $14.8 \%$ & $8.8 \%$ & $8.4 \%$ & $46.6 \%$ & $39.1 \%$ & $49.5 \%$ & $15.3 \%$ & $30.8 \%$ & $41.0 \%$ & $20.8 \%$ \\
\hline 9 & $10.4 \%$ & $12.4 \%$ & $11.4 \%$ & $13.6 \%$ & $24.6 \%$ & $9.0 \%$ & $13.2 \%$ & $17.4 \%$ & $22.1 \%$ & $14.8 \%$ & $44.2 \%$ & $25.8 \%$ & $22.7 \%$ & $10.2 \%$ & $18.3 \%$ & $23.8 \%$ & $16.2 \%$ \\
\hline 10 & $13.1 \%$ & $15.3 \%$ & $12.8 \%$ & $16.3 \%$ & $27.8 \%$ & $17.3 \%$ & $19.3 \%$ & $17.2 \%$ & $23.8 \%$ & $17.1 \%$ & $37.2 \%$ & $25.7 \%$ & $21.4 \%$ & $12.4 \%$ & $19.4 \%$ & $23.6 \%$ & $18.0 \%$ \\
\hline 11 & $10.7 \%$ & $12.1 \%$ & $10.7 \%$ & $12.8 \%$ & $23.2 \%$ & $10.1 \%$ & $13.4 \%$ & $17.4 \%$ & $21.6 \%$ & $14.1 \%$ & $20.5 \%$ & $32.5 \%$ & $27.4 \%$ & $33.3 \%$ & $25.7 \%$ & $30.9 \%$ & $17.2 \%$ \\
\hline 12 & $15.5 \%$ & $15.2 \%$ & $12.5 \%$ & $14.9 \%$ & $28.0 \%$ & $16.1 \%$ & $20.3 \%$ & $18.0 \%$ & $25.7 \%$ & $17.3 \%$ & $22.1 \%$ & $32.7 \%$ & $23.5 \%$ & $29.9 \%$ & $23.3 \%$ & $34.0 \%$ & $18.9 \%$ \\
\hline 13 & $10.4 \%$ & $9.7 \%$ & $6.6 \%$ & $10.4 \%$ & $17.3 \%$ & $9.7 \%$ & $10.6 \%$ & $18.1 \%$ & $16.4 \%$ & $10.5 \%$ & $12.7 \%$ & $25.6 \%$ & $30.5 \%$ & $3.7 \%$ & $26.5 \%$ & $24.3 \%$ & $15.4 \%$ \\
\hline 14 & $22.3 \%$ & $23.9 \%$ & $17.7 \%$ & $23.8 \%$ & $38.0 \%$ & $26.7 \%$ & $28.8 \%$ & $18.5 \%$ & $37.2 \%$ & $24.8 \%$ & $54.9 \%$ & $19.6 \%$ & $20.8 \%$ & $17.9 \%$ & $12.0 \%$ & $13.3 \%$ & $22.2 \%$ \\
\hline 15 & $16.9 \%$ & $15.6 \%$ & $21.4 \%$ & $15.7 \%$ & $15.1 \%$ & $12.3 \%$ & $14.7 \%$ & $27.6 \%$ & $14.6 \%$ & $15.5 \%$ & $23.3 \%$ & $41.6 \%$ & $47.8 \%$ & $32.4 \%$ & $36.5 \%$ & $97.3 \%$ & $25.6 \%$ \\
\hline 16 & $21.5 \%$ & $25.1 \%$ & $27.2 \%$ & $25.5 \%$ & $21.7 \%$ & $25.8 \%$ & $20.7 \%$ & $27.6 \%$ & $21.5 \%$ & $22.6 \%$ & $58.5 \%$ & $73.0 \%$ & $60.4 \%$ & $64.4 \%$ & $52.1 \%$ & $49.4 \%$ & $36.5 \%$ \\
\hline 17 & $11.3 \%$ & $14.3 \%$ & $15.5 \%$ & $14.2 \%$ & $16.0 \%$ & $16.1 \%$ & $13.3 \%$ & $17.3 \%$ & $15.4 \%$ & $13.4 \%$ & $37.7 \%$ & $55.7 \%$ & $43.0 \%$ & $47.6 \%$ & $42.1 \%$ & $41.3 \%$ & $25.0 \%$ \\
\hline 18 & $14.9 \%$ & $13.4 \%$ & $14.9 \%$ & $14.5 \%$ & $18.4 \%$ & $23.2 \%$ & $17.5 \%$ & $22.6 \%$ & $17.0 \%$ & $13.5 \%$ & $33.3 \%$ & $50.9 \%$ & $45.4 \%$ & $40.8 \%$ & $35.2 \%$ & $31.6 \%$ & $26.0 \%$ \\
\hline 19 & $8.0 \%$ & $7.3 \%$ & $4.8 \%$ & $8.5 \%$ & $18.8 \%$ & $26.3 \%$ & $15.8 \%$ & $13.9 \%$ & $16.1 \%$ & $8.9 \%$ & $22.0 \%$ & $45.3 \%$ & $31.3 \%$ & $10.8 \%$ & $28.8 \%$ & $20.1 \%$ & $18.4 \%$ \\
\hline 20 & $14.1 \%$ & $10.3 \%$ & $11.3 \%$ & $8.5 \%$ & $13.9 \%$ & $28.1 \%$ & $16.8 \%$ & $18.3 \%$ & $12.1 \%$ & $9.9 \%$ & $30.5 \%$ & $40.0 \%$ & $44.6 \%$ & $37.3 \%$ & $38.2 \%$ & $35.3 \%$ & $24.4 \%$ \\
\hline 21 & $18.4 \%$ & $19.9 \%$ & $23.3 \%$ & $18.9 \%$ & $8.7 \%$ & $29.4 \%$ & $14.6 \%$ & $22.5 \%$ & $11.6 \%$ & $15.6 \%$ & $47.4 \%$ & $69.8 \%$ & $62.0 \%$ & $60.2 \%$ & $56.2 \%$ & $45.7 \%$ & $34.4 \%$ \\
\hline 22 & $12.6 \%$ & $14.1 \%$ & $14.6 \%$ & $14.8 \%$ & $18.7 \%$ & $9.9 \%$ & $12.2 \%$ & $16.2 \%$ & $17.4 \%$ & $13.8 \%$ & $28.7 \%$ & $44.5 \%$ & $32.6 \%$ & $35.0 \%$ & $27.9 \%$ & $32.9 \%$ & $20.2 \%$ \\
\hline 23 & $9.4 \%$ & $13.1 \%$ & $13.7 \%$ & $12.8 \%$ & $13.5 \%$ & $14.0 \%$ & $7.9 \%$ & $16.3 \%$ & $14.6 \%$ & $11.6 \%$ & $29.0 \%$ & $47.1 \%$ & $36.4 \%$ & $26.9 \%$ & $24.2 \%$ & $91.0 \%$ & $22.1 \%$ \\
\hline 24 & $11.2 \%$ & $14.7 \%$ & $15.5 \%$ & $14.2 \%$ & $16.7 \%$ & $15.3 \%$ & $11.3 \%$ & $9.1 \%$ & $15.9 \%$ & $13.7 \%$ & $42.7 \%$ & $55.8 \%$ & $43.4 \%$ & $52.3 \%$ & $45.2 \%$ & $37.4 \%$ & $25.6 \%$ \\
\hline 25 & $15.7 \%$ & $22.8 \%$ & $27.5 \%$ & $27.1 \%$ & $8.9 \%$ & $20.8 \%$ & $14.0 \%$ & $22.8 \%$ & $7.1 \%$ & $16.3 \%$ & $46.7 \%$ & $99.8 \%$ & $68.7 \%$ & $86.0 \%$ & $44.7 \%$ & $62.1 \%$ & $35.2 \%$ \\
\hline 26 & $12.0 \%$ & $11.3 \%$ & $13.4 \%$ & $10.1 \%$ & $14.1 \%$ & $17.2 \%$ & $12.3 \%$ & $16.6 \%$ & $11.6 \%$ & $9.0 \%$ & $39.2 \%$ & $52.0 \%$ & $45.9 \%$ & $54.7 \%$ & $42.7 \%$ & $39.3 \%$ & $24.9 \%$ \\
\hline 27 & $32.9 \%$ & $36.5 \%$ & $26.1 \%$ & $25.2 \%$ & $63.8 \%$ & $55.7 \%$ & $55.8 \%$ & $44.2 \%$ & $58.6 \%$ & $41.0 \%$ & $6.8 \%$ & $12.7 \%$ & $8.2 \%$ & $29.7 \%$ & $12.1 \%$ & $94.6 \%$ & $33.4 \%$ \\
\hline 28 & $18.9 \%$ & $20.9 \%$ & $18.7 \%$ & $23.4 \%$ & $30.4 \%$ & $13.6 \%$ & $20.5 \%$ & $17.1 \%$ & $27.1 \%$ & $22.8 \%$ & $12.4 \%$ & $9.7 \%$ & $13.3 \%$ & $12.5 \%$ & $14.5 \%$ & $11.8 \%$ & $17.1 \%$ \\
\hline 29 & $14.7 \%$ & $21.1 \%$ & $18.2 \%$ & $24.4 \%$ & $32.6 \%$ & $11.3 \%$ & $23.6 \%$ & $19.3 \%$ & $25.7 \%$ & $23.4 \%$ & $11.7 \%$ & $10.0 \%$ & $6.8 \%$ & $12.8 \%$ & $7.4 \%$ & $10.8 \%$ & $16.1 \%$ \\
\hline 30 & $11.2 \%$ & $12.5 \%$ & $11.8 \%$ & $15.8 \%$ & $15.6 \%$ & $34.6 \%$ & $10.8 \%$ & $16.2 \%$ & $16.2 \%$ & $13.9 \%$ & $16.2 \%$ & $26.6 \%$ & $29.6 \%$ & $9.6 \%$ & $16.0 \%$ & $15.3 \%$ & $19.8 \%$ \\
\hline 31 & $16.1 \%$ & $23.0 \%$ & $21.0 \%$ & $28.2 \%$ & $35.0 \%$ & $8.4 \%$ & $28.5 \%$ & $23.1 \%$ & $24.6 \%$ & $25.3 \%$ & $10.4 \%$ & $9.8 \%$ & $6.8 \%$ & $14.5 \%$ & $6.4 \%$ & $11.5 \%$ & $16.8 \%$ \\
\hline 32 & $18.7 \%$ & $18.5 \%$ & $15.8 \%$ & $22.0 \%$ & $31.1 \%$ & $19.9 \%$ & $25.9 \%$ & $18.4 \%$ & $26.9 \%$ & $20.6 \%$ & $18.7 \%$ & $10.9 \%$ & $5.9 \%$ & $13.0 \%$ & $7.9 \%$ & $7.3 \%$ & $17.8 \%$ \\
\hline rerage & $15.4 \%$ & $16.7 \%$ & $16.2 \%$ & $17.2 \%$ & $23.8 \%$ & $20.0 \%$ & $19.2 \%$ & $20.1 \%$ & $21.4 \%$ & $17.3 \%$ & $31.1 \%$ & $35.9 \%$ & $31.6 \%$ & $27.3 \%$ & $25.9 \%$ & $36.5 \%$ & - \\
\hline
\end{tabular}

${ }^{\text {a }}$ Site ID in first row represent for the sites (sources site) that provide data constructing SVM model for solar radiation estimation at the site (estimation site) indicated by site ID in the first column. 
Table 4

Correlation coefficients between RMSE of SVM and distance between source site and estimation site, altitude of source site, and difference of maximum (RMSE $\mathrm{tmax}_{\mathrm{tma}}$ ), and minimum ( $\left.\mathrm{RMSE}_{\mathrm{tmin}}\right)$ air temperature between source site and estimation site.

\begin{tabular}{clcccc}
\hline Site ID & Site name & Distance & Altitude & RMSE $_{\text {tmin }}$ & RMSE $_{\text {tmax }}$ \\
\hline 1 & Lvshi & 0.113 & 0.347 & -0.124 & -0.080 \\
2 & Shanghai & 0.297 & 0.347 & $0.556^{* *}$ & $0.459^{* *}$ \\
3 & Nanjing & $0.434^{*}$ & $0.647^{* *}$ & $0.692^{* *}$ & $0.694^{* *}$ \\
4 & Hangzhou & $0.431^{*}$ & $0.689^{* *}$ & $0.689^{* *}$ & $0.704^{* *}$ \\
5 & Hongjia & $0.469^{* *}$ & $0.637^{* *}$ & $0.775^{* *}$ & $0.762^{* *}$ \\
6 & Fuzhou & 0.317 & $0.465^{* *}$ & 0.160 & 0.179 \\
7 & Jianou & 0.328 & $0.400^{*}$ & 0.161 & 0.165 \\
8 & Tunxi & $0.396^{*}$ & $0.574^{* *}$ & $0.587^{* *}$ & $0.613^{* *}$ \\
9 & Hefei & $0.406^{*}$ & $0.636^{* *}$ & $0.708^{* *}$ & $0.681^{* *}$ \\
10 & Gushi & $0.427^{*}$ & $0.569^{* *}$ & $0.703^{* *}$ & $0.670^{* *}$ \\
11 & Wuhang & $0.724^{* *}$ & $0.531^{* *}$ & $0.387^{*}$ & $0.481^{* *}$ \\
12 & Nanchang & $0.659^{* *}$ & $0.465^{* *}$ & 0.323 & $0.436^{*}$ \\
13 & Ganzhou & 0.239 & 0.265 & 0.167 & 0.162 \\
14 & Shangtou & 0.132 & $0.390^{*}$ & $0.430^{*}$ & $0.556^{* *}$ \\
15 & Guangzhou & $0.451^{* *}$ & $0.426^{*}$ & 0.167 & 0.044 \\
16 & Changling & $0.550^{* *}$ & $0.477^{*}$ & $0.460^{* *}$ & $0.574^{* *}$ \\
17 & Changsha & $0.685^{* *}$ & $0.543^{* *}$ & $0.499^{* *}$ & $0.576^{* *}$ \\
18 & Yichang & $0.582^{* *}$ & 0.269 & $0.367^{*}$ & $0.419^{*}$ \\
19 & Nanyang & $0.547^{* *}$ & 0.193 & $0.446^{*}$ & $0.378^{* *}$ \\
20 & Ankang & $0.546^{* *}$ & 0.214 & $0.373^{* *}$ & $0.382^{*}$ \\
21 & Jishou & $0.581^{* *}$ & 0.302 & $0.371^{*}$ & $0.428^{*}$ \\
22 & Guiling & $0.398^{*}$ & $0.453^{* *}$ & 0.286 & $0.409^{* *}$ \\
23 & Nanling & 0.061 & $0.376^{*}$ & 0.131 & 0.049 \\
24 & Guiyang & 0.201 & $0.363^{*}$ & 0.333 & 0.263 \\
25 & Chongqing & $0.386^{*}$ & 0.330 & 0.221 & $0.416^{*}$ \\
26 & Mianyang & 0.291 & $0.373^{*}$ & $0.379^{* *}$ & $0.460^{* *}$ \\
27 & Emeishan & -0.154 & $0.400^{*}$ & $0.434^{*}$ & 0.345 \\
28 & Mengzhi & 0.076 & 0.339 & -0.147 & -0.011 \\
29 & Kunming & 0.063 & $0.370^{*}$ & 0.122 & 0.206 \\
30 & Panzhihua & 0.181 & -0.106 & -0.069 & -0.151 \\
31 & Lijiang & 0.049 & 0.348 & 0.197 & 0.171 \\
32 & Tengchong & 0.218 & $0.367^{*}$ & $0.492^{* *}$ & 0.299 \\
& & & &
\end{tabular}

* Significant at 0.05 significance level.

* Significant at 0.01 significance level.

are (1) to investigate the feasibility of SVM model developed at source site for estimation of solar radiation at estimation site; and (2) to explore influencing factors of source SVM model accuracy.

\section{Materials and methods}

\subsection{Study area}

China's subtropical zone (Fig. 1) is located in the east of the Eurasian continent, on the south of the Huai River-Qin Mountains which separate the subtropical zone from the warm temperature zone, to the east of the Qinghai-Tibet plateau which separates the subtropical zone from the Qinghai-Tibet plateau climate zone, bordering the west coast of the Pacific Ocean. It covers an area of $240 \times 10^{4} \mathrm{~km}^{2}$, occupying about $25 \%$ area of mainland China [56]. The elevation generally decreases from west to east. The western part of the area is dominated by Yunnan-Guizhou Plateau with an average altitude exceeding $2000 \mathrm{~m}$, the northern part is dominated by Middle-Lower Yangtze River Plain, and the southern part is dominated by rolling hills. Due to the prevailing of the East Asian monsoon [57], China's subtropical zone has unique climate features characterized by four distinct seasons with a hot, humid summer, and warm to cool winter, and plenty of precipitation with a highly pronounced summer peak, which differs significantly from other regions at the same latitude where the climates are dominated by Mediterranean and Semi-desert/desert climates [58]. The topography, soil and climate contributed to the complex ecosystem and abundant biodiversity [56], and hence the ecological, agricultural and the associated researches are extensively carried out in this area [59-61].

\subsection{Site and data}

All the meteorological stations (32 sites) in studying area with long-term available solar radiation and air temperature data are used. The mapping of stations roughly range from $22^{\circ}$ to $33^{\circ} \mathrm{N}$ (latitude), from $98^{\circ}$ to $122^{\circ} \mathrm{E}$ (longitude), and from 1 to $3047 \mathrm{~m}$ altitude. Table 1 shows the detailed information of the studied meteorological stations. For each station, 15 years monthly data covering the period between 1994 and 2008 were obtained from the National Meteorological Information Center (NMIC), China Meteorological Administration (CMA). Global solar radiation $\left(\mathrm{MJ} \mathrm{m}^{-2}\right)$ is measured by pyranometer, and air temperatures $\left({ }^{\circ} \mathrm{C}\right)$ including maximum and minimum temperatures are measured by mercury and alcohol thermometers [62]. All the instruments are calibrated periodically and all the measurements are made following the guide of World Meteorological Organization [63]. Preliminary quality control tests were conducted by the suppliers. We further checked the data and removed records according to the following criterions: (a) actual solar radiation larger than the extra-terrestrial solar radiation, and (b) minimum air temperature larger than maximum air temperature.

\subsection{Extraction of geographic information}

The distance between source and estimation site is calculated by measurement tool in ARCGIS software. Firstly, Table 1 was converted into point shapefile with geographic coordinate system. The shapefile illustrates the spatial distribution of the studied meteorological stations positioned by longitude and latitude in Table 1. Secondly, the point shapefile was projected to Albers projection with central meridian of 105 , first standard parallel of 25 and second standard parallel of 47 . Then the distance between two sites can be easily measured in ARCGIS software. Other geographic information including longitude, latitude and altitude of the site can be directly obtained from Table 1 .

\subsection{Support vector machine}

SVM is a supervised learning model with associated learning algorithm that analyzes data and recognizes patterns, widely used in classification [44], regression [45,46] and forecasting [47]. The advantages of SVM can be summarized as follows: SVM is based on statistical learning theory and principle of structural risk minimization, which attempts to minimize an upper bound of generalization error rather than minimize the local training error. This is the most significant difference from the commonly used principle of empirical risk minimization $[64,65]$. SVM estimates the regression using a set of kernel functions which are defined in a high dimensional feature space. SVM delivers a unique solution, since the optimality problem is convex. This is an advantage compared to Neural Networks, which have multiple solutions associated with local minima [66]. A brief introduction to the theory of SVM was presented in our preliminary work [3]. More detailed information can be found in Vapnik $[64,65]$.

One of the key tasks in estimation of solar radiation using SVM is the selection of the input attributes. Our preliminary work [3] suggested that SVM model using maximum and minimum air temperature significantly outperformed other models. Consequently, they are used as input attributes, and the ratio of actual radiation to extra-terrestrial radiation is used as output attribute (label). Extra-terrestrial solar radiation dependents on solar constant, the relative distance between the sun and the earth, the latitude, the sunset hour angle and the solar declination, it is calculated using the equations detailed by Allen et al. [51].

For each estimation site, the data from each individual source site (32 sites) are used as training data to build the SVM model. 

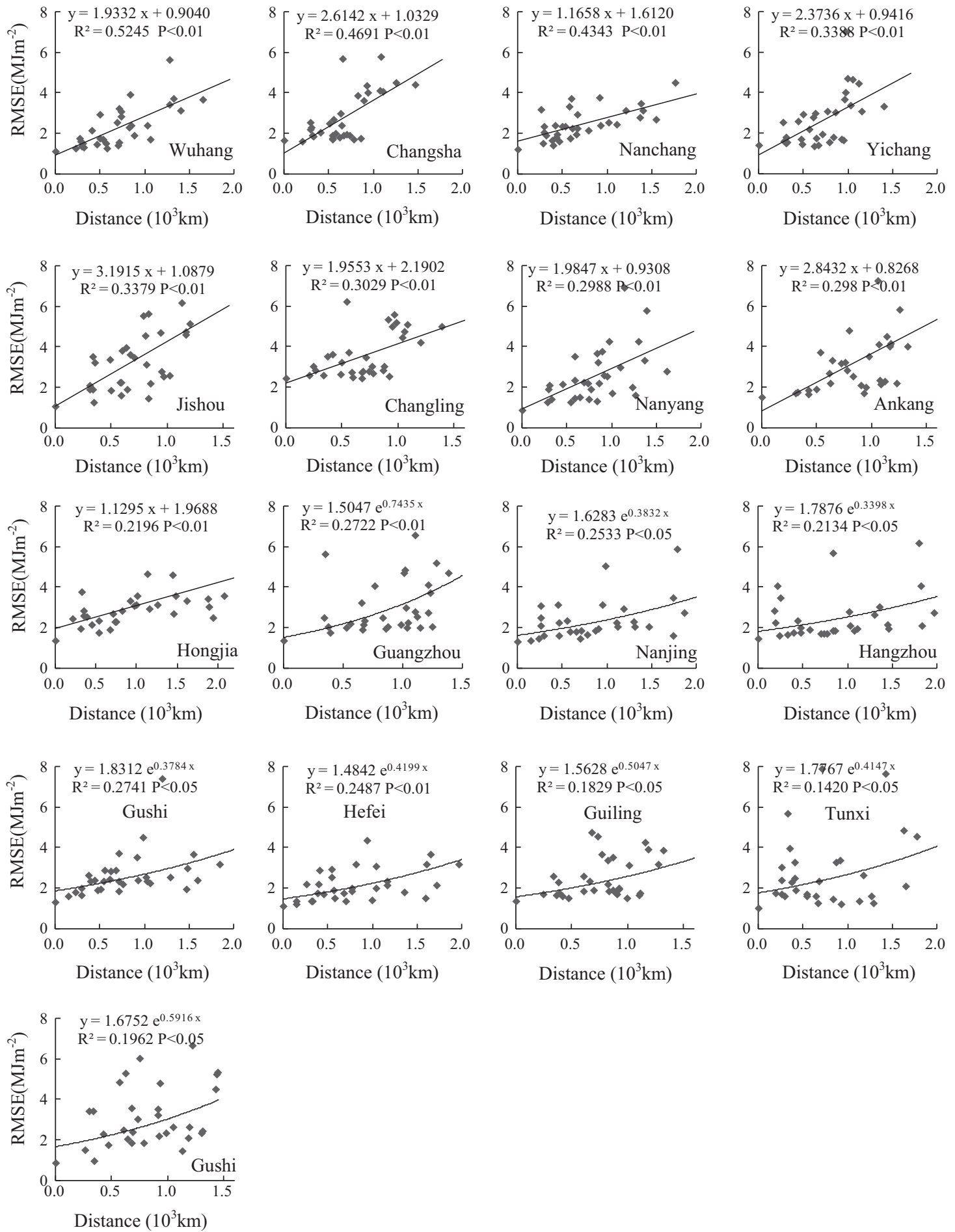

Fig. 2. Relation between RMSE of SVM $\left(\mathrm{MJ} \mathrm{m}^{-2}\right)$ and the distance between the estimation site and source site.

The trained SVM models are used to estimate solar radiation at the estimation site, then the estimations are tested against the measurements. The procedure is repeated 32 times for each estimation site, each time using a different SVM model with training data from different source site.

The SVM models are implemented by LIBSVM which is an integrated software for support vector classification and regression [67]. The procedures include data scaling, training and testing, more detailed information can be found in Hsu et al. [68].

\subsection{Performance criteria}

Determination correlation $\left(R^{2}\right)$, root mean square error (RMSE) and mean absolute percentage error (MAPE, \%) are used to evaluate the performance of SVM models. The metric $R^{2}$ varying between 0 and 1 is adopted to measure the strength of a relationship, higher the value, stronger the relationship. In addition, $t$-test is carried out to determine the model's statistical significance which is expressed in probability levels ( $p$ value), the smaller the $p$-level, the more 


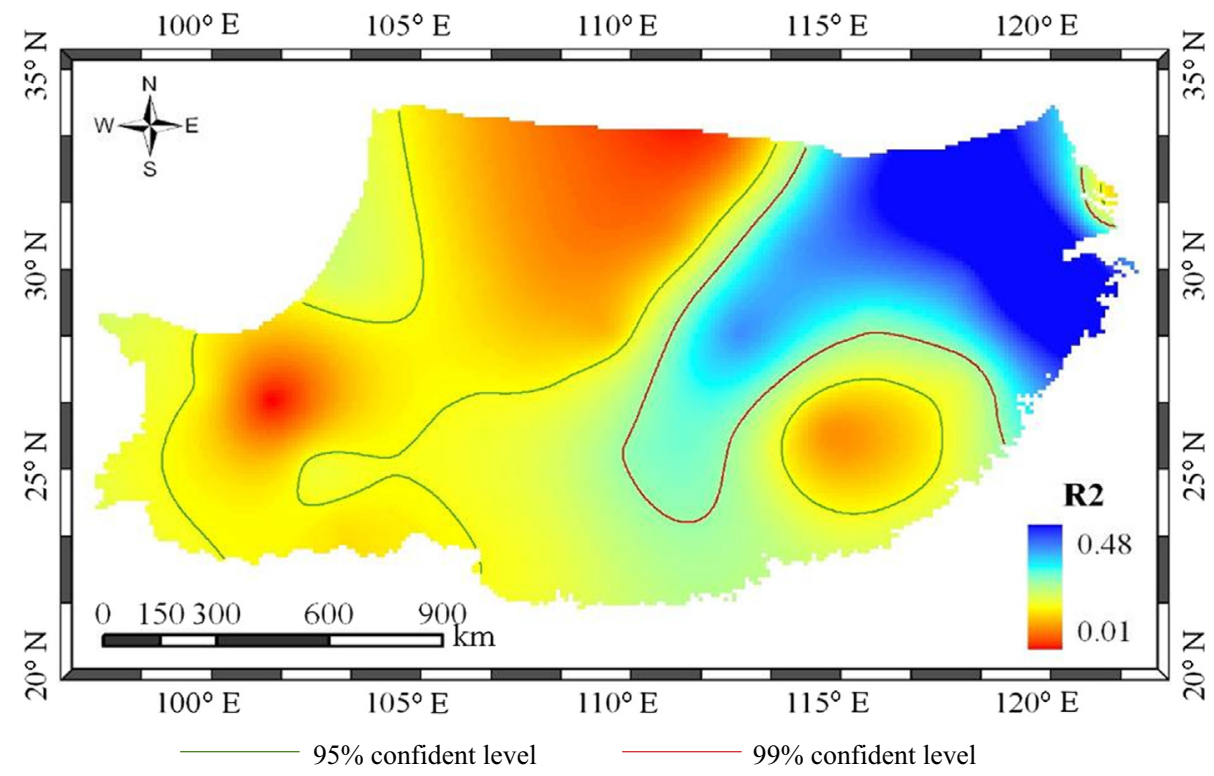

Fig. 3. Spatial variation of determination correlation between RMSE $\left(\mathrm{MJ} \mathrm{m}^{-2}\right)$ of SVM and distance between source site and estimation site.

significant the relationship. RMSE provides information on the short term performance of the correlations by allowing a term by term comparison of the actual deviation between the estimated and measured values. MAPE is a dimensionless index allowing comparisons among a range of different model responses regardless of units, it can give a general judgement of the model performance at different station. Lower values of RMSE, and MAPE indicate a better performance. RMSE and MAPE are calculated by the following equations.

$R M S E=\sqrt{\frac{\sum_{i=1}^{n}\left(y_{i}-\hat{y}_{i}\right)^{2}}{n}}$

$M A P E=\frac{1}{n}\left|\frac{y_{i}-\hat{y}_{i}}{y_{i}}\right|$

where $n, y$, and $\hat{y}$ represent the number of testing data, the observed value, and the estimated value, respectively.

\section{Results}

\subsection{Performances of SVM models}

Performances of SVM models are presented in Tables 2 and 3, in which the error indicators (RMSE and MAPE) are produced by SVM model developed at source site in first row for solar radiation estimation at estimation site in first column, and those in diagonal (hereafter RMSE $E_{\text {dia }}$, MAPE $_{\text {dia }}$ ) are produced by SVM developed using the estimation site's own data. As it can be seen, SVM shows good generalization with RMSE $_{\text {dia }}$ varying between 0.84 and $2.66 \mathrm{MJ} \mathrm{m}^{-2}$ (average 1.41), and $\mathrm{MAPE}_{\mathrm{dia}}$ varying between $4.8 \%$ and $19 \%$ (average $8.6 \%$ ). This confirms the result in our previous case study [3], and further proves that SVM is a promising alternative for predicting solar radiation at the sites where the measurements of air temperatures are available.

In the case of unavailable solar radiation for constructing SVM model, solar radiation could be well estimated by SVM model developed at another site. For example, at Panzhihua (ID 30) SVM model developed at Nanjing (ID 3), and Hangzhou (ID 4) produce RMSE of 2.09 , and $2.11 \mathrm{MJ} \mathrm{m}^{-2}$, respectively, which are similar to $1.97 \mathrm{MJ} \mathrm{m}^{-2}$ driven by its own data. This has potential attraction in generating solar radiation data for studies in agriculture, environment and ecology, especially for the large, remote areas where ground measurements of solar radiation are difficult, even impossible. Suppose that if the difference of RMSE between source and estimation site is less than $20 \%$, the source site would be acceptable for the estimation site. The source sites are classified into 3 categories, while -1 indicates the source site that has lower RMSE than the estimation site, 1 indicates the difference of RMSE between source and estimation sites less than $20 \%$, and 2 indicates other sites. The acceptable source sites have been highlighted for each estimation site (Table 2). The sites (e.g. Fuzhou (ID 6), Changling (ID 16)) with higher number of category -1 and 1 have more alternative substitution sites. It is interesting that at some estimation sites SVM model developed at source sites (indicated by -1) even returns lower RMSE and MAPE than those driven by their own data. One possible reason may be that the time period for developing SVM model may have a role affecting the air temperature-radiation relationship, and thus climate change and consequent non-stationarities in time series of air temperature and radiation may affect the model performance. This climate change and non-stationarities trends are reported globally and regionally [69]. Some works pointed out that different periods for developing models showed some degree of climate change effect which was site-specific [70]. Therefore, it would be significant to investigate further in detail how climate change affects model accuracy, and this is especially important for reconstructing historical radiation data, but is beyond the objective of this study.

The average RMSE and MAPE in each row reflect the possibility of solar radiation estimation by SVM model developed at other sites. Lower values of average RMSE and MAPE (e.g. Ganzhou (ID 13)), Wuhang (11)) means that solar radiation are relatively easier to be estimated by SVM model developed at other sites. The average RMSE and MAPE in each column indicate the overall representativeness of source site in estimation of solar radiation for other sites. The sites (e.g. Nanjing (ID 3), Hangzhou (ID 4)) with lower values of RMSE and MAPE are relatively more universal. Nevertheless, for the studied sites in this work, the acceptable sources sites and the transferable SVM models should be guided by the results (Tables 2 and 3 ). While for other sites, the factors influencing the transferability of a source model need to be investigated. 
(a)

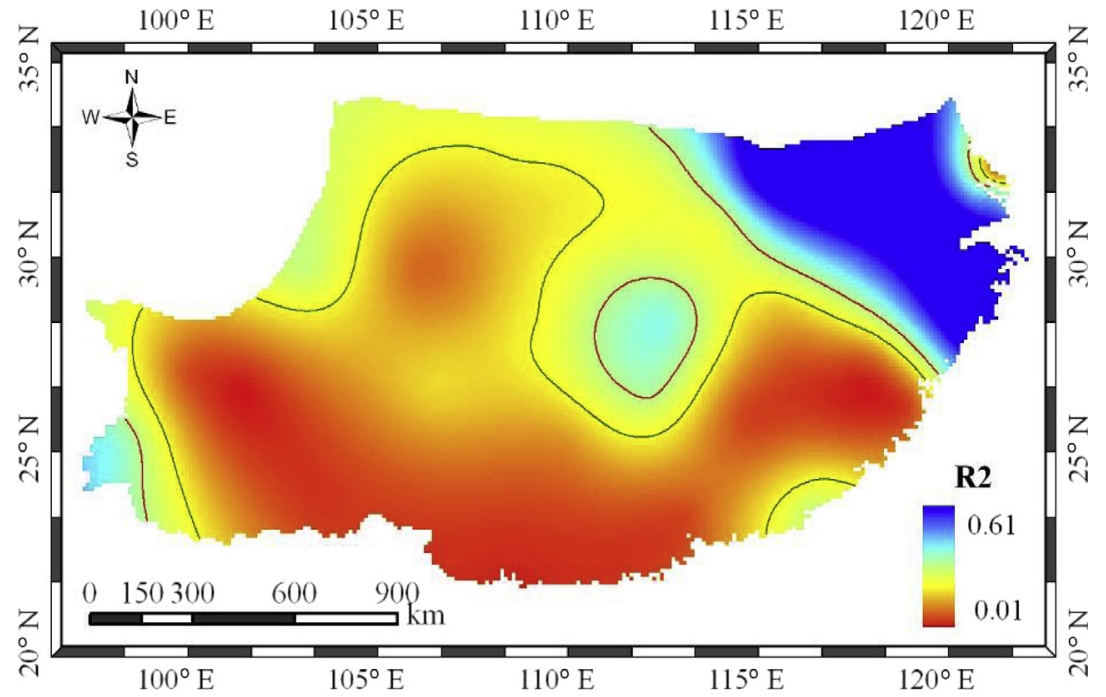

(b)

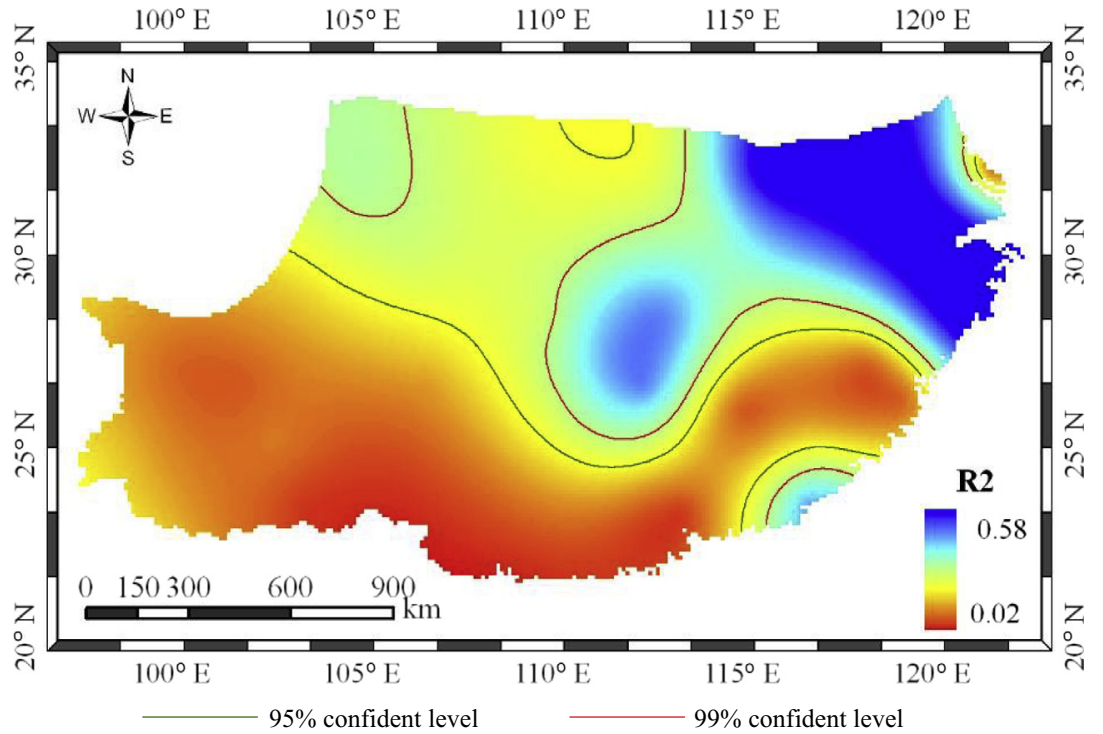

Fig. 4. Spatial variation of determination correlation between $\operatorname{RMSE}\left(\mathrm{MJ} \mathrm{m}^{-2}\right)$ of $S V M$ and $\operatorname{RMSE}_{\mathrm{tmin}}\left({ }^{\circ} \mathrm{C}\right)(\mathrm{a})$, and $\mathrm{RMSE}_{\mathrm{tmax}}\left({ }^{\circ} \mathrm{C}\right)(\mathrm{b})$.

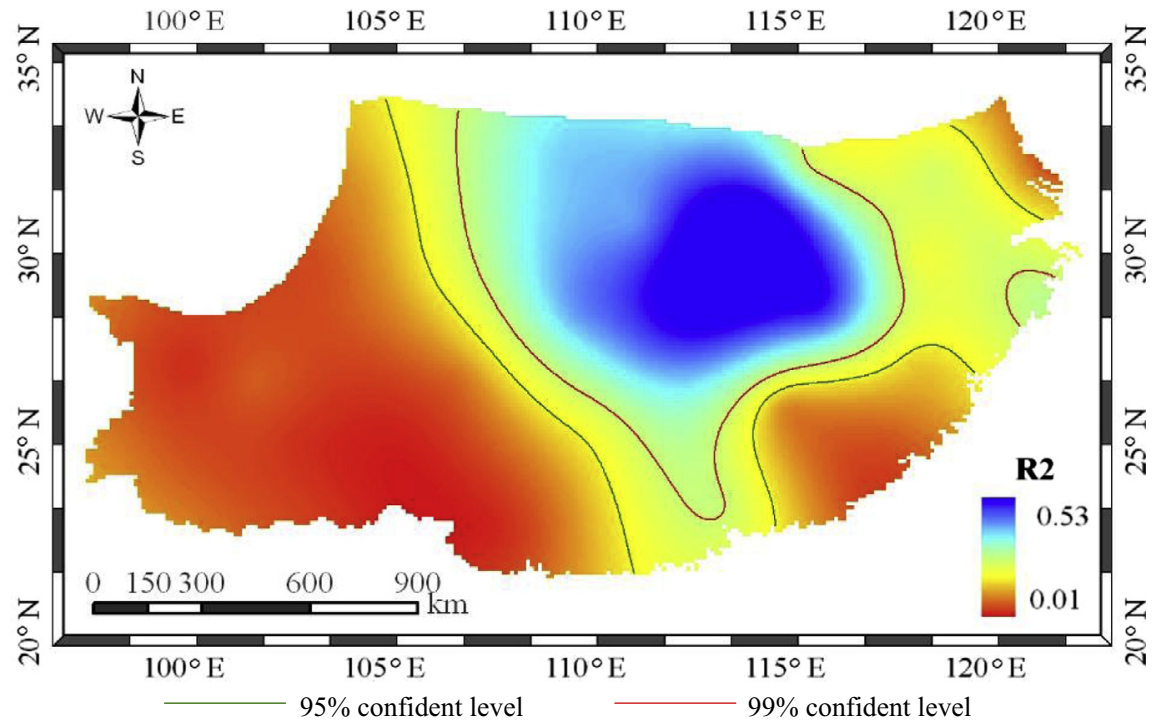

Fig. 5. Spatial variation of determination correlation between RMSE $\left(\mathrm{MJ} \mathrm{m}^{-2}\right)$ of SVM and altitude of source site. 

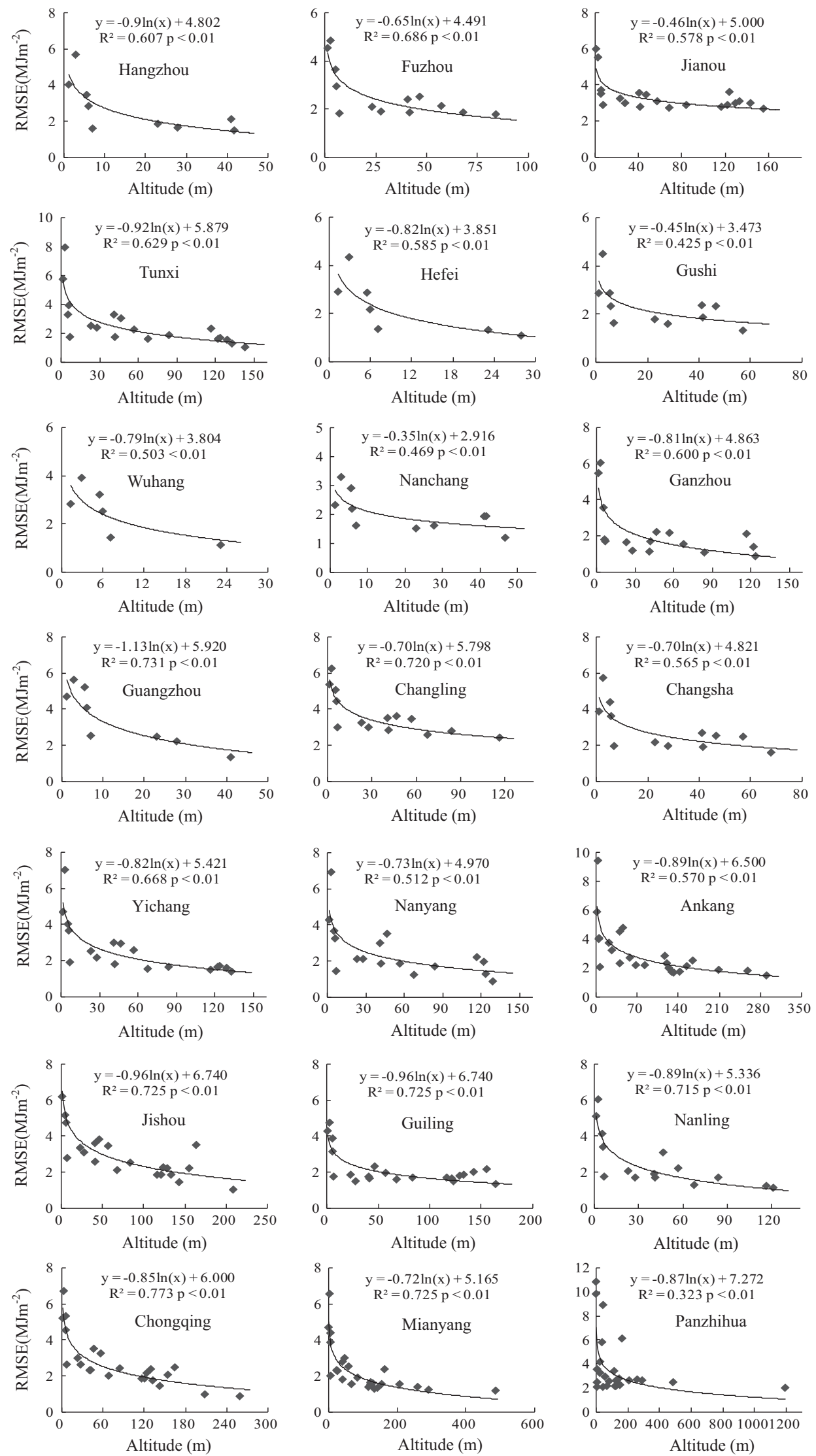

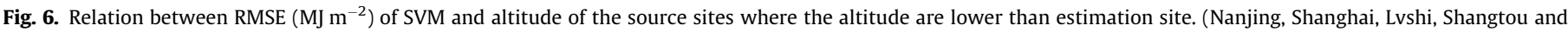
Hongjia are not analyzed as the number of source sites where the altitudes are lower than them is less than 5). 


\subsection{Analyses of factors influencing the transferability of source model}

At each estimation site RMSE and MAPE vary considerably from source site to site. Consequently, the relation between model accuracy and distance (hereafter distance) between source and estimation site, altitude and air temperatures of source site are investigated. The results are summarized in Table 4.

At most estimation sites distance has significant effect on accuracy of source SVM models. Correlation analysis shows that RMSE correlate significantly with distance at 17 estimation sites, with the correlation coefficients varying between $0.386(p<0.05)$ and 0.724 $(p<0.01)$. Fig. 2 plots RMSE against distance for those 17 sites. At 9 sites RMSE increase as a linear function of distance, while at the remaining 8 sites, RMSE increase with the increase in distance in an exponential manner. These are different from the results on RMSE of directly replacing solar radiation by those from nearby sites versus distance for other places. For example, in Canada, Hunt et al. [1] reported that RMSE increased with increase in distance in a curvilinear manner. Nonhebel [71] and Trnka et al. [28] also explored the possible replacement of missing radiation data by measurements from a nearby station, and revealed similar RMSE versus distance relationship as that reported by Hunt et al. [1]. The most probable reason for the difference lies in different topography and climate. However, no significant relationship is found between RMSE and distance at the remaining 15 sites (Table 4), this is consistent with the result reported by Liu and Scott [21] for Australia.

Fig. 3 shows the spatial distribution of $R^{2}$ between RMSE of SVM and distance. The map is generated by Kriging interpolation which allows us to obtain spatially continuous data from values of surrounding site based on a semivariogram model [72,73]. As can be seen, $R^{2}$ varies greatly from regions to regions. Higher values are presented in northeastern region of the studying area, while lower values in northern central region and southwestern region. The northeastern region is a part of Lower Yangtze River Plain where the topography and climate condition are similar. The significantly relationship between RMSE and distance increases the reliability and confidence of source SVM model from nearby stations. While the weak correlations in northern central region and southwestern region limit the possibility of solar radiation estimation using data from nearby stations, this may be due to the complex topography and complicated climate. These results reveal that solar radiation could be well estimated by SVM model developed using data from nearby locations with similar climatic conditions and topography.

When temperature data at estimation site are replaced by the data at source sites, RMSE are also calculated to measure the difference of temperature between two sites. The correlation analysis shows that RMSE of SVM (Table 4) correlate significantly with RMSE of minimum temperature difference (hereafter RMSE $_{\text {tmin }}$ ) at 18 estimation sites, and that show significant correlations with RMSE of maximum temperature difference (hereafter RMSE tmax $_{\text {) }}$ at 19 estimation sites (Table 4). The spatial distribution (Fig. 4) shows that $R^{2}$ between RMSE of SVM and RMSE $\mathrm{t}_{\mathrm{tmin}}$, and $\mathrm{RMSE}_{\mathrm{tmax}}$ present similar spatial variations which generally decrease from northeast toward southwest. The topography and climate conditions in the northeastern region are quite similar. The higher correlations in this region imply that the sites with lower temperature differences tend to have similar temperature-radiation relationships, and thus the solar radiation could be estimated by SVM model developed using the data from other sites with lower temperature differences.

At most sites altitude of source sites has significant effect on accuracy of source SVM models. RMSE correlate significantly with altitude at 19 estimation sites, with the correlation coefficients varying between $0.363(p<0.05)$ and $0.689(p<0.01)$. The spatial distribution (Fig. 5) of $R^{2}$ between RMSE and altitude shows that higher values of $R^{2}$ are presented in central region. The significant correlations generally indicate that accuracy of SVM decrease with the increase in altitude of source sites. While lower $R^{2}$ in the southwest region implies that the temperature-solar radiation relationship in this plateau varies greatly from altitude to altitude.

Further investigation reveals interesting results on relationship between accuracy of SVM and altitude of the source sites where the altitude are lower than that of estimation site. For the estimation sites where the altitude are lower than $1200 \mathrm{~m}$, RMSE show significantly logarithm relationship with the altitude of source sites, as it can be seen from Fig. 6, RMSE decrease with the increase in altitude in a logarithm manner, with $R^{2}$ varying between $0.323(p<0.01)$ and $0.774(p<0.01)$. In contrast, for the estimation sites where the altitude are higher than $1200 \mathrm{~m}$, RMSE show significantly linearly relationship with the altitude of the source sites where the altitude are higher than $200 \mathrm{~m}$ but lower than that of the estimation site, as it can be seen from Fig. 7, RMSE decrease with
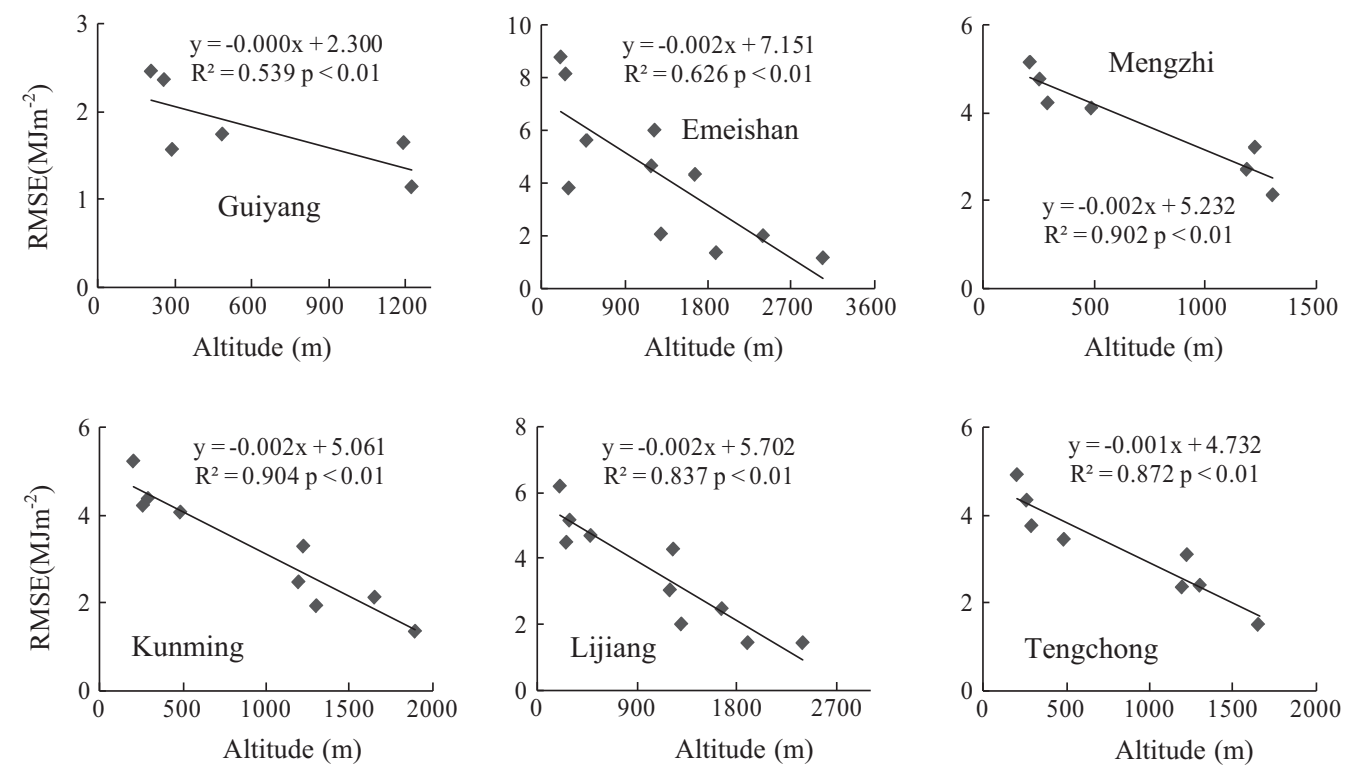

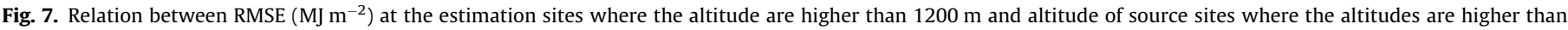
$200 \mathrm{~m}$ but lower than that of the estimation site. 
the increase in altitude in a linearly manner, with $R^{2}$ varying between $0.539(p<0.01)$ and $0.905(p<0.01)$. Two different RMSE versus altitude relationships indicate that temperature-solar radiation relationships differ greatly from plateau to other regions. The higher correlations suggest that the sites with the similar altitude tend to have similar temperature-solar radiation relationship, and reveal that solar radiation at one site could be estimated by SVM model developed using the data from other sites where the altitude are similar but lower than that of the estimation site.

\section{Conclusions and future prospect}

The transferability of SVM model for solar radiation estimation is investigated in subtropical zone in China. Results suggest that solar radiation at one site could be well estimated by SVM model developed at another sites. The accuracy of source SVM model is affected by distance and temperature differences between estimation and source site, and altitude of source site. Higher correlations between RMSE of SVM and distance, and temperature differences are observed in northeastern region with similar climatic conditions and topography, increasing the reliability and confidence of radiation estimation by SVM model developed using data from nearby stations. While lower correlations are presented in southwest plateau region due to the complex topography and complicated climate. When altitude of estimation site is lower than $1200 \mathrm{~m}$, RMSE show logarithm relationship with altitude of source sites where the altitude are lower than that of estimation site, when altitude is higher than $1200 \mathrm{~m}$, RMSE show linearly relationship with the altitude of source sites where the altitude are higher than $200 \mathrm{~m}$ but lower than that of the estimation site. The results suggest that radiation at one site could be also estimated by SVM model developed at other sites with similar but lower altitude.

The findings have implications relative to the deployment of resource in agricultural, environmental, and ecological researches. Since many researchers are making efforts to measure radiation at the sites that are often not visited. Because of cost of measuring equipments, instrument malfunctioning, sensor degradation and calibration, the data from such sites may be of questionable quality. It may be preferable, therefore, for agricultural, environmental, and ecological researchers to concentrate their efforts to measure radiation at representative sites or source sites where the data can be used to develop a transferable SVM model for other sites. For the studied sites in China's subtropical zone, selection of a suitable source sites can be guided by the results in this work, while for other sites in studying area, a strategy for selecting a suitable source sites is presented: Firstly, the source sites should be restricted in the area with similar climatic conditions and topography to the estimation sites, then the distance should be considered, the source sites with shorter distance to the estimation site is preferred, lastly, the altitude should be considered, the source sites with similar but lower altitude than the estimation site is superior to other sites.

Admittedly, the $R^{2}$ are relatively low compared to those of the site-specific models reported by other researchers. This may be attributed to that only air temperatures of each individual source site are used as training data. Besides air temperature, sunshine duration, relative humidity, precipitation and atmospheric pressure are also routinely measured at most meteorological stations. Many works showed that models using these meteorological variables in combination with air temperature significantly outperformed models using air temperature alone [13,52,55]. Thus, future work will investigate the potential of source SVM models using more combination of meteorological variables or combining data sets from several similar source sites to increase the $R^{2}$ and reliability of the new proposed method.

\section{Acknowledgments}

The work was supported by Chongqing Science and Technology Key project (cstc2012ggB20001), West Light Foundation of the Chinese Academy of Sciences, National Natural Science Foundation of China (41401051) and three gorges follow-up planning foundation. We thank the National Meteorological Information Center, China Meteorological Administration for providing the long-term data records. Many thanks go to the anonymous reviewers for the comments on the manuscript.

\section{References}

[1] Hunt LA, Kuchar L, Swanton CJ. Estimation of solar radiation for use in crop modelling. Agric For Meteorol 1998;91:293-300.

[2] Yorukoglu M, Celik AN. A critical review on the estimation of daily global solar radiation from sunshine duration. Energy Convers Manage 2006; $47(15): 2441-50$.

[3] Chen JL, Liu HB, Wu W, Xie DT. Estimation of monthly solar radiation from measured temperatures using support vector machines - a case study. Renew Energy 2011;36(1):413-20.

[4] Ball RA, Purcell LC, Carey SK. Evaluation of solar radiation prediction models in North America. Agron J 2004;96:391-7.

[5] Garcia AGY, Hoogenboom G. Evaluation of an improved daily solar radiation generator for the southeastern USA. Clim Res 2005;29:91-102.

[6] Rivington M, Bellocchi G, Matthews KB, Buchan K. Evaluation of three model estimations of solar radiation at 24 UK stations. Agric For Meteorol 2005;132:228-43.

[7] Robaa SM. Validation of the existing models for estimating global solar radiation over Egypt. Energy Convers Manage 2009;50(1):184-93.

[8] Polo J, Zarzalejo LF, Cony M, Navarro AA, Marchante R, Martin L, et al. Solar radiation estimations over India using Meteosat satellite images. Sol Energy 2011;85:2395-406.

[9] Notton G, Cristofari C, Muselli M, Poggi P. Calculation on an hourly basis of solar diffuse irradiations from global data for horizontal surfaces in Ajaccio. Energy Convers Manage 2004;45:2849-66.

[10] Jacovides CP, Tymvios FS, Assimakopoulos VD, Kaltsounides NA. Comparative study of various correlations in estimating hourly diffuse fraction of global solar radiation. Renew Energy 2006;31(15):2492-504.

[11] Bortolini M, Gamberi M, Graziani A, Manzini R, Mora C. Multi-location model for the estimation of the horizontal daily diffuse fraction of solar radiation in Europe. Energy Convers Manage 2013;67:208-16.

[12] Chen JL, Li GS. Assessing the potential of support vector machine for estimating daily solar radiation using sunshine duration. Energy Convers Manage 2013;75:311-8.

[13] Chen JL, Li GS. Estimation of monthly average daily solar radiation from measured meteorological data in Yangtze River Basin in China. Int J Climatol 2013:33:487-98.

[14] Chen JL, Li GS. Parameterization and mapping of solar radiation in data sparse regions. Asia-Pacific J Atmos Sci 2012;48(4):423-31.

[15] Hargreaves GH, Samani ZA. Estimating potential evapotranspiration. J Irrig Drain Div 1982;108:225-30.

[16] Bristow KL, Campbell GS. On the relationship between incoming solar radiation and daily maximum and minimum temperature. Agric For Meteorol 1984:31:159-66.

[17] Demirhan H, Turhan M, Mustafa A. Statistical comparison of global solar radiation estimation models over Turkey. Energy Convers Manage 2013;68:141-8.

[18] Zhao N, Zeng XF, Han SM. Solar radiation estimation using sunshine hour and air pollution index in China. Energy Convers Manage 2013;76:846-51.

[19] De JR, Stewart DW. Estimating global solar radiation from common meteorological observations in western Canada. Can J Plant Sci 1993;73:509-18.

[20] Thornton PE, Running SW. An improved algorithm for estimating daily solar radiation from measurements of temperature, humidity, and precipitation. Agric For Meteorol 1999;93:211-28.

[21] Liu DL, Scott BJ. Estimation of solar radiation in Australia from rainfall and temperature observations. Agric For Meteorol 2001;106:41-59.

[22] Wu J, Chan CK. Prediction of hourly solar radiation with multi-model framework. Energy Convers Manage 2013;76:347-55.

[23] Chen RS, Lu SH, Kang E, Yang JP, Ji XB. Estimating daily global radiation using two types of revised models in China. Energy Convers Manage 2006;47(7):865-78.

[24] Weiss A, Hays CJ, Hu Q, Easterling WE. Incorporating bias error in calculating solar irradiance: implications for crop yield simulations. Agron 2001:93:1321-6.

[25] Abraha MG, Savage MJ. Comparison of estimates of daily solar radiation from air temperature range for application in crop simulations. Agric For Meteorol 2008;148:401-16.

[26] Winslow JC, Hunt JR, Piper SC. A globally applicable model of daily solar irradiance estimated from air temperature and precipitation data. Ecol Model 2001;143:227-43. 
[27] Mahmood R, Hubbard KG. Effect of time and temperature observation and estimation of daily solar irradiance for the northern Great Plains, USA. Agron J 2002;94:723-33.

[28] Trnka M, Zalud Z, Eitzinger J, Dubrovský M. Global solar radiation in Centra European lowlands estimated by various empirical formulae. Agric For Meteorol 2005;131:54-76.

[29] Gueymard C, Jidra P, Eatrada CV. A critical look at recent interpretations of the Ångström approach and its future in global solar irradiation prediction. Sol Energy 1995;54:357-63.

[30] Rehman S, Mohandes M. Artificial neural network estimation of global solar radiation using air temperature and relative humidity. Energy Policy 2008;36:571-6.

[31] Benghanem M, Mellit A, Alamri SN. ANN-based modelling and estimation of daily global solar radiation data: a case study. Energy Convers Manage 2009;50(7):1644-55.

[32] Martí P, Gasque M. Ancillary data supply strategies for improvement of temperature-based ETo ANN-models. Agric Water Manage 2010;97(7):939-55.

[33] Koca A, Oztop HF, Varol Y, Koca GO. Estimation of solar radiation using artificial neural networks with different input parameters for Mediterranean region of Anatolia in Turkey. Exp Syst Appl 2011;38:8756-62.

[34] Zarzo M, Martí P. Modeling the variability of solar radiation data among weather stations by means of principal component analysis. Appl Energy 2011;88(8):2775-84

[35] Solmaz O, Ozgoren M. Prediction of hourly solar radiation in six provinces in Turkey by Artificial Neural Networks. J Energy Eng 2012;138(4):194-204.

[36] Ozgoren M, Bilgili M, Sahin B. Estimation of global solar radiation using ANN over Turkey. Exp Syst Appl 2012;39(5):5043-51.

[37] Solmaz O, Ozgoren M, Aksoy MH. Hourly cooling load prediction of a vehicle in the southern region of Turkey by Artificial Neural Network. Energy Convers Manage 2014;82:177-87.

[38] Shiri J, Nazemi AH, Sadraddini AA, Landeras G, Kisi O, Fard AF, et al. Global cross-station assessment of neuro-fuzzy models for estimating daily reference evapotranspiration. J Hydrol 2013;480:46-57.

[39] Shiri J, Sadraddini AA, Nazemi AH, Kisi O, Landeras G, Fard AF, et al Generalizability of Gene Expression Programming-based approaches for estimating daily reference evapotranspiration in coastal stations of Iran. Hydrol 2014;508:1-11.

[40] Lee SW, Verri A. Support vector machines for computer vision and pattern recognition. Int J Pattern Recogn Artif Intell 2003;3:331-2.

[41] Lu WZ, Wang W. Potential assessment of the support vector machine method in forecasting ambient air pollutant trends. Chemosphere 2005;59:693-701.

[42] Stanislaw O, Konrad G. Forecasting of the daily meteorological pollution using wavelets and support vector machine. Eng Appl Artif Intell 2007:20:745-55.

[43] Tirusew A, Mariush K, Mac M, Abedalrazq K. Multi-time scale stream flow predictions: the support vector machines approach. J Hydrol 2006;318:7-16.

[44] Huang C, Davis LS, Townshend JRG. An assessment of support vector machines for land cover classification. Int J Rem Sens 2002;23:725-49.

[45] Vapnik VN, Golowich SE, Smola AJ. Support vector method for function approximation, regression estimation and signal processing. In: Mozer $\mathrm{M}$ Jordan M, Petsche T, editors. Advances in neural information processing systems. Cambridge (MA): MIT Press; 1996.

[46] Collobert R, Bengio S. SVMTorch: support vector machines for large-scale regression problems. J Mach Learn Res 2001;1:143-60.

[47] Tay FEH, Cao LJ. Application of support vector machines in financial time series forecasting. Int J Manage Sci 2001;29:309-17.

[48] Dibike BY, Velickov S, Solomatine D, Abbott MB. Model induction with support vector machines: introduction and applications. J Comput Civ Eng 2001;15(3):208-16.

[49] Liong SY, Sivapragasam C. Flood stage forecasting with support vector machines. J Am Water Resour Assoc 2002;38:173-86.
[50] Kecman V. Support vector machines-an introduction. In: Wang L, editor. Support vector machines: theory and applications. New York: Springer-Verlag; 2005. p. $1-48$.

[51] Allen RG, Pereira LS, Raes D, Smith M. Crop evapotranspiration-guidelines for computing crop water requirements. FAO Irrigation and Drainage Paper 56. Rome: Food and Agriculture Organization of the United Nations. 1998.

[52] Chen RS, Ersi K, Yang JP, Lu SH, Zhao WZ. Validation of five global radiation models with measured daily data in China. Energy Convers Manage 2004:45:1759-69.

[53] Li MF, Liu HB, Guo PT, Wu W. Estimation of daily solar radiation from routinely observed meteorological data in Chongqing, China. Energy Convers Manage 2010;51(12):2575-9.

[54] Wu W, Liu HB. Assessment of monthly solar radiation estimates using support vector machines and air temperatures. Int J Climatol 2012;32(2):274-85.

[55] Wu GF, Liu YL, Wang TJ. Methods and strategy for modeling daily global solar radiation with measured meteorological data - a case study in Nanchang station, China. Energy Convers Manage 2007;48:2447-52.

[56] Yang QY, Zheng D, Wu SH. On subtropical zone of China. J Subtrop Resour Environ 2006:1(1):1-10.

[57] Wang B, Huang F, Wu Z, Yang J, Fu X, Kikuchi K. Multi-scale climate variability of the South China Sea monsoon: a review. Dynam Atmos Oceans 2009;47(1):15-37.

[58] Zhang J, Ge Y, Chang J, Jiang B, Jiang H, Peng C, et al. Carbon storage by ecological service forests in Zhejiang Province, subtropical China. For Ecol Manage 2007;245(1):64-75.

[59] Cao MK, Tao B, Li KR, Shao XM, Stephen DP. Interannual variation in terrestrial ecosystem carbon fluxes in China from 1981 to 1998. Acta Bot Sin 2003;45(5):552-60.

[60] You L, Rosegrant MW, Wood S, Sun D. Impact of growing season temperature on wheat productivity in China. Agric For Meteorol 2009;149(6):1009-14.

[61] Waha K, Van Bussel LG, Müller C, Bondeau A. Climate-driven simulation of global crop sowing dates. Global Ecol Biogeogr 2012;21(2):247-59.

[62] China Meteorological Administration (CMA). Specifications for surface meteorological observation. QX/T45-2007; 1979. p. 187.

[63] World Meteorological Organization (WMO). Guide to the Meteorological Instruments and Methods of Observation. WMO-8; 1983. p. 517.

[64] Vapnik VN. The nature of statistical learning theory. New York: Springer Verlag; 1995.

[65] Vapnik VN. Statistical learning theory. New York: Wiley; 1998.

[66] Auria L, Moro RA. Support vector machines as a technique for solvency analysis. Berlin: German Institute for Economic Research; 2008. No. 811.

[67] Chang C, Lin C. LIBSVM: a library for support vector machines; 2001. <http:// www.csie.ntu.edu.tw/ cjlin/libsvm> [Software].

[68] Hsu CW, Chang CC, Lin CJ. A practical guide to support vector classification. <http://www.csie.ntu.edu.tw/ cjlin/papers/guide/guide.pdf>; 2003.

[69] Liu BH, Xu M, Henderson M, Qi Y, Li YQ. Taking China’s temperature: daily range, warming trends, and regional variations, 1955-2000. J Clim 2004:17:4453-62.

[70] Liu X, Mei X, Li Y, Wang Q Jensen JR, Zhang Y, et al. Evaluation of temperaturebased global solar radiation models in China. Agric For Meteorol 2009;149(9):1433-46.

[71] Nonhebel S. The importance of weather data in crop growth simulation models and assessment of climate change effects. PhD thesis. Wageningen Agriculture University; 1993. P. 144.

[72] Merino GG, Jones D, Stooksbury DE, Hubbard KG. Determination of semivariogram models to krige hourly and daily solar irradiance in western Nebraska. J Appl Meteorol 2002;40:1085-94.

[73] Alsamamra H, Ruiz-Arias JA, Pozo-Vázquez D, Tovar-Pescador J. A comparative study of ordinary and residual kriging techniques for mapping global solar radiation over southern Spain. Agric For Meteorol 2009;149:1343-57. 